\title{
Diagnosing a Strong-Fault Model by Conflict and Consistency
}

\author{
Wenfeng Zhang ${ }^{1}{ }^{(0)}$, Qi Zhao ${ }^{1}{ }^{(1)}$, Hongbo Zhao ${ }^{1, *}{ }^{(\mathbb{D})}$, Gan Zhou ${ }^{2}$ and Wenquan Feng ${ }^{1}$ \\ 1 Electronic and Information Engineering, Beihang University, Beijing 100191, China; \\ zhangwenfeng42153@buaa.edu.cn (W.Z.); zhaoqi@buaa.edu.cn (Q.Z.); buaafwq@buaa.edu.cn (W.F.) \\ 2 The 6th Research Institute of China Electronics Corporation, Beijing 100083, China; zhouganterry@163.com \\ * Correspondence: bhzhb@buaa.edu.cn; Tel.: +86-10-8231-7210
}

Received: 28 February 2018; Accepted: 26 March 2018; Published: 29 March 2018

\begin{abstract}
The diagnosis method for a weak-fault model with only normal behaviors of each component has evolved over decades. However, many systems now demand a strong-fault models, the fault modes of which have specific behaviors as well. It is difficult to diagnose a strong-fault model due to its non-monotonicity. Currently, diagnosis methods usually employ conflicts to isolate possible fault and the process can be expedited when some observed output is consistent with the model's prediction where the consistency indicates probably normal components. This paper solves the problem of efficiently diagnosing a strong-fault model by proposing a novel Logic-based Truth Maintenance System (LTMS) with two search approaches based on conflict and consistency. At the beginning, the original a strong-fault model is encoded by Boolean variables and converted into Conjunctive Normal Form (CNF). Then the proposed LTMS is employed to reason over CNF and find multiple minimal conflicts and maximal consistencies when there exists fault. The search approaches offer the best candidate efficiency based on the reasoning result until the diagnosis results are obtained. The completeness, coverage, correctness and complexity of the proposals are analyzed theoretically to show their strength and weakness. Finally, the proposed approaches are demonstrated by applying them to a real-world domain - the heat control unit of a spacecraft-where the proposed methods are significantly better than best first and conflict directly with $\mathrm{A}^{*}$ search methods.
\end{abstract}

Keywords: fault diagnosis; model-based diagnosis; truth maintenance system; conflict directed A*; a strong-fault model

\section{Introduction}

As human beings rely on sophisticated systems more than ever, keeping their operation safe is quite significant for the environment, lives and property. Although the systems usually undergo rigorous tests before deployment, they may still be faulty because of wear, degradation and unexpected harm. Diagnosing their faults in time can stop the faults from spreading to other parts, resulting in irretrievable disaster [1-3].

Numerous diagnosis methods have been proposed in the last few decades [4-8] and most of them come from two domains: Artificial Intelligence (AI) [9] and Control Theory [3]. The methods can also be divided into two classes based on where system information comes from: the model-based method $[10,11]$ and the data-driven method $[12,13]$.

This paper only focuses on the Model-Based Diagnosis (MBD) proposed by Reiter [9] in the DX (Diagnosis from first principle) community, where MBD adopts reasoning methods in AI to diagnose by discrete model, MBD is composed of modeling (for example, first order logic) and reasoning. The reasoning can be further separated into two steps: conflict recognition and hitting sets generation. The hitting sets of all minimal conflicts have been proven to be the diagnoses for a weak-fault model 
theoretically [9]. Kleer and Williams [14,15] also independently found the theory which, however, focuses on getting all the conflicts by Assumption-based Truth Maintenance System (ATMS) [16,17]. The validity of the theory has been verified by the General Diagnosis Engine (GDE) [15,18]. But GDE consumes plenty of computation resources when diagnosing complex systems due to "combinatorial explosion."

Practically speaking, only a few diagnosis solutions with a high weight are useful, which means not all the candidates should be analyzed. There are two types of famous approximation approaches to ignoring low weight candidates: a stochastic approach and a search approach.

The most famous stochastic approach is Safari $[19,20]$ proposed by Feldman. Safari first assumes that all faults exist. Then it selects one fault randomly, sets it as normal (flip the state value) and obtains a new candidate. If the candidate causes conflicts, the flip is retreated and selects a fault randomly again. If there is no conflict, the random selection is applied to the new candidate. A diagnosis solution (the latest candidate) is obtained if there are conflicts in the last $n$ (a preset parameter) random selection. Safari is optimal in probability for a weak-fault model and more efficient compared with search-based diagnosis methods. However, the optimality of Safari is not ensured for a strong-fault model [20]. So, this method is not chosen as the basis of this paper.

Search approaches usually obey the idea of "guess and verify": they employ a search method to guess a most probable candidate first and then verify if the candidate is a valid diagnosis. Williams [18] and Kurien [21] proposed the Best-First (BF) diagnosis method, which utilized a search algorithm to find the candidate with maximal priori probability first and then checks whether there are conflicts between the system model, observation and the candidate. A diagnosis is obtained when a candidate with no conflict is found. Further, Williams proposed the Conflict Directed $A^{*}\left(\mathrm{CDA}^{*}\right)$ [22] search method which selected the best candidates based on a priori probability and verified them as well. But CDA* caches conflict sets recognized by the Logic-based Truth Maintenance System (LTMS) [23] and the verification for some candidates is skipped if they contain some conflicts. In this way, CDA* avoids much unnecessary computation and saves a lot of time. As one of the most important factors for diagnosis, conflict is widely studied. Stern explored the duality between conflict and diagnosis [24] and some divide and conquer methods [25-27] are proposed to reduce the cost to find conflicts.

The key techniques in the search approaches discussed above are how to utilize a priori information and conflict, which are also important research points in the DX community recently. However, much research concentrates too much on conflict to notice that even the consistency between model prediction and observation contains diagnosis information: the part in the candidate, which causes the model prediction, is probably right. Besides, traditional methods are proposed for a weak-fault model with only one normal mode and one unknown fault mode. The correctness is not ensured for a strong-fault [19] model in which fault modes are described with specific behaviors.

This paper studies conflict and consistency directed diagnosis methods for a strong-fault model comprehensively and has the following novelties: (1) A new conception, consistency (formally defined in Section 3.2.2), is proposed to boost diagnose; (2) a novel LTMS is proposed to obtain multiple conflicts and consistencies in one reasoning process; (3) proposes a more accurate distribution model about candidate and observation; (4) based on the distribution in (3), proposes two $A^{*}$ search-based algorithms that make full use of conflicts and consistencies to get single-diagnosis and multiple-diagnosis respectively.

The rest of this paper is organized as follows: Section 2 introduces the formal definitions and methods about system model, diagnosis problem and the conflict directed $\mathrm{A}^{*}$ for a weak-fault model; Section 3 discusses the proposed method in detail; Section 4 analyzes the algorithms proposed comprehensively; Section 5 demonstrates the proposed methods by a real-word model: a heat control unit of a spacecraft; The last section presents the conclusion and future work.

\section{Theoretical Background}

This section introduces the basic definitions and methods about DX diagnosis methods. 


\subsection{Discrete Model}

In DX, models are usually logic propositions in which variables are discrete: Boolean or Enumerative. Based on the description manner of discrete variables, the models are divided into weak-fault and a strong-fault models.

\subsubsection{Logic Based Diagnosis Model}

Logic (such as first order logic) is a powerful language to describe complex behaviors and used in many famous diagnosis systems such as GDE and Sherlock [14].

Formally, a diagnosis model (DM) is defined by tuple (1).

$$
<S V, S D, M D>
$$

where:

- $\quad S V$ (system variable) is the finite set of system variables. The set can be partitioned into mode variables $(M O D E)$, observation variables (OBS) and inner variables (INNER). The possible behavioral modes of each component are defined by the mode variable. Observation variables means their values can be obtained from sensors and in this paper (and other most cases) they are assumed to be perfectly correct. And inner variable is all other variables.

- $S D$ (system description) is a finite set of logic propositions which are constraints over $S V$. Usually $S D$ is represented by CNF or Negative Normal Form (NNF) [28].

- $\quad M D$ (mode distribution) is the prior probability distribution of mode variables.

Usually, models for components are first built and then are conjoined as (2) where $C$ is a finite set of logic propositions, which describes how subsystems connect to each other.

$$
\begin{aligned}
& S V=\cup S V_{i} \\
& S D=\left(\cup S D_{i}\right) \cup C \\
& M D=\cup M D_{i}
\end{aligned}
$$

\subsubsection{Weak-Fault Model and Strong-Fault Model}

The model which has only one normal mode and an unknown fault mode is called a weak-fault model. On the contrary, a model with specific fault modes is a strong-fault model. In other words, given the inputs, the outputs are determined for each fault mode in a strong-fault model.

$$
\begin{aligned}
& S V=\{\text { mode }, \text { in } 1, \text { in } 2, \text { out }\}, \text { mode } \in\{\text { normal }, \text { fault }\}, \text { in } 1, \text { in } 2, \text { out } \in\{\text { true }, \text { false }\} \\
& S D=\{(\text { mode }==\text { normal }) \Rightarrow(\text { in } 1 \text { and in } 2==\text { out })\} \\
& M D=\{P(\text { mode }=\text { normal })=0.99, P(\text { mode }=\text { fault })=0.01\}
\end{aligned}
$$

The weak-fault model of the adder in Figure 1 is shown in (3). In this weak-fault model, the system has only two modes: normal and fault. When the mode is normal, out must be equal to "in 1 and in $2 . "$ When the mode is fault, there is no constraint over inputs and output. Sometimes a weak-fault model uses a bool variable as the mode variable instead.

$$
\begin{aligned}
S V= & \{\text { mode }, \text { in } 1, \text { in } 2, \text { out }\}, \text { mode } \in\{\text { normal }, \text { or_faul }\}, \text { in } 1, \text { in } 2, \text { out } \in\{\text { true }, \text { false }\} \\
S D=\quad & (\text { mode }==\text { normal }) \Rightarrow(\text { in } 1 \text { and in } 2==\text { out }), \\
& (\text { mode }==\text { or_fault }) \Rightarrow(\text { in } 1 \text { or in } 2==\text { out })\} \\
M D= & \{P(\text { mode }=\text { normal })=0.99, P(\text { mode }=\text { or_fault })=0.01\}
\end{aligned}
$$

The strong-fault model of the adder in Figure 1 is shown in (4). Compared with model (3), the strong-fault model has a fault mode or fault, in which out is equal to "in 1 or in 2 " by mistake. 
The most significant difference between the two models is that the strong-fault model specifies the behaviors even for fault mode.

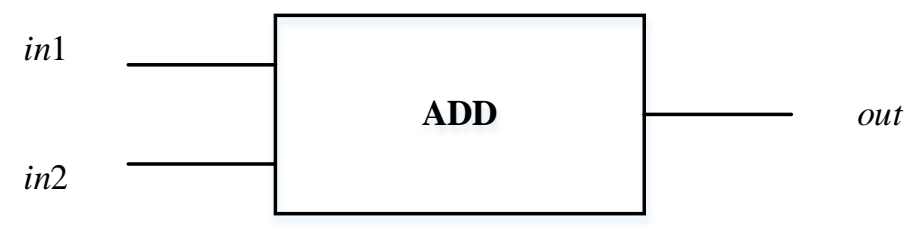

Figure 1. Adder.

Diagnosing a strong-fault model is quite difficult because of the non-monotonicity. In a weak-fault model, setting a component as a fault will never cause a conflict because there is no constraint in fault mode. The famous Safari [20] utilizes this feature and starts diagnosis by assuming that all components are faulty. In a strong-fault model, if a mode's behavior is not consistent with the observation, anyone in the rest may be available.

\subsection{Diagnosis Problem}

Given a DM, fault diagnosis is to find diagnosis solutions (diagnosis for short) that explain the health states of systems. This subsection gives some definitions formally. They are a little different from the definitions in other literatures $[20,29]$ to be more suitable for a strong-fault model.

A Diagnosis Problem (DP) is defined by tuple (5).

$$
<D M, \text { obs }>
$$

where:

- $D M$ is the diagnosis model of a system.

- obs is the assignment set of observation variables.

\subsubsection{Diagnosis}

A diagnosis $\omega_{s}$ is an assignment set of mode variables that is consistent with the system model and the observation as (6). The diagnosis discussed in this paper is static.

$$
S D \cup o b s \cup \omega_{s} \mid \neq \perp
$$

\subsubsection{Preferred Diagnosis}

The definition of diagnosis above only describes what a diagnosis is but what is a good diagnosis is not given. Usually, diagnosis is evaluated by posterior probability $P$ (mode $\mid$ obs) which can be decomposed by Bayes rule as in Equation (7).

$$
P(\text { mode } \mid \text { obs })=\frac{P(\text { mode }) P(\text { obs } \mid \text { mode })}{P(\text { obs })}
$$

where:

- $\quad P($ mode $)$ is the priori probability of mode.

- $\quad P(o b s \mid$ mode $)$ is the conditional probability of observation obs given mode. For simplification, in most cases, $P$ (obs $\mid$ mode) is restricted in $\{0,1\}$ (when there exist conflicts, $P($ obs $\mid$ mode $)=0$; when there is no conflict, $P($ obs $\mid$ mode $)=1$ ) which means the diagnosis is evaluated by priori probability instead of posterior probability. However, the simplification ignores system structure and implies that the existence of conflict is the only flag of if the candidate mode is right, which means that 
both false alarm and missing alarm are zero. This paper proposes a new scheme to compute it more accurate.

- $\quad P(o b s)$ is the marginal probability of observation obs and there is no need to compute it because it is a normalization factor.

Most approximation diagnosis methods [19] design a routine based on (7) to search for the best candidate and then check the consistency between the candidate and observation until the diagnosis (consistent candidate) is found. To the author's knowledge, the top two famous methods are Best First $\mathrm{A}^{*}\left(\mathrm{BFA}^{*}\right)$ search and Conflict Directed $\mathrm{A}^{*}\left(\mathrm{CDA}^{*}\right)$ search. BFA* uses priori information $(P($ mode $))$ only and $\mathrm{CDA}^{*}$ uses both priori $(P($ mode $))$ and conflicts information (parts of $P\left(\right.$ obs $\mid$ mode) ). As $\mathrm{CDA}^{*}$ is the one of the state of art methods and the basis of our approaches, it is introduced briefly in the next subsection.

\subsection{Conflict Directed $A^{*}$ Search for Weak Model}

The search space size of a diagnosis problem is $d^{n}$, where $d$ is the domain's average size of each mode variable and $n$ is the size of set MODE. It is too costly to find all the diagnoses. However, most diagnoses are insignificant because of their low probabilities. $\mathrm{CDA}^{*}$ employs the theorem about diagnosis and conflict to avoid testing unnecessary mode assignments.

A conflict $c$ is an assignment set of mode variables which satisfies that

$$
S D \cup o b s \cup c \mid=\perp
$$

Without confusion, we also call the difference between observed value and predicted value a conflict (or between predicted values).

If $C$ is a set of sets $c_{1}, c_{2} \ldots c_{n}$, set $h$ is a hitting set of $C$ if

$$
\forall c \in C, h \cap c \neq \varnothing
$$

If $C$ includes all the conflict sets, $c$ is sure to be a diagnosis.

To find the best candidate effectively, the conflict directed $\mathrm{A}^{*}$ method develops a novel framework shown in Figure 2. The $\mathrm{A}^{*}$ search finds a best candidate based on priori probability and the conflicts obtained till now. LTSM checks the consistence of the candidate, the system model and observation. If it is consistent, the candidate is a diagnosis and added into the diagnosis set. Otherwise, LTMS finds the minimal conflicts, adds them into conflict set and a new iteration starts. Williams introduces two distinct search algorithms for a single-diagnosis and multiple diagnoses.

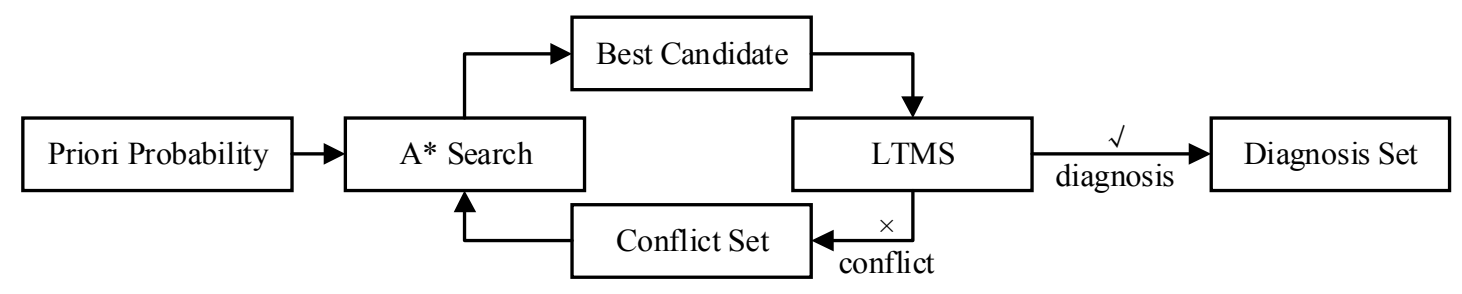

Figure 2. Framework of Conflict Directed $A^{*}$.

\subsubsection{Single-Diagnosis}

To find only one diagnosis, Williams generates a hitting set of current conflicts as the new candidate to be checked. To get the preferred hitting set efficiently, $\mathrm{CDA}^{*}$ generates constituent kernels (CK) of each conflict first and then generates kernels that resolve all the conflicts.

A constituent kernel of conflict $c$ is an individual assignment that resolves $c$.

A kernel of the set $C$ including all conflict sets is the minimal set that resolves all conflicts in $C$ (no subset of kernel resolves all conflicts). 
The $A^{*}$ search is employed to find a specific kernel. Figure 3 shows a search tree created by CDA* where blank nodes are invalid nodes and grey nodes are valid nodes. The two blank nodes are not kernels because they are not minimal and the two nodes in the right are not extended because they are able to resolve both conflicts.

When there is no conflict in the beginning, the candidate assumes that all components are normal. When conflicts are found by LTMS, the conflict manager generates the next best kernel by resolving all the conflicts and gets the next best candidate by setting all other components as normal. To be brief, Figure 3 evaluates candidates by the number of fault component and kernels are best candidates. In practice, kernels are also evaluated by probability.

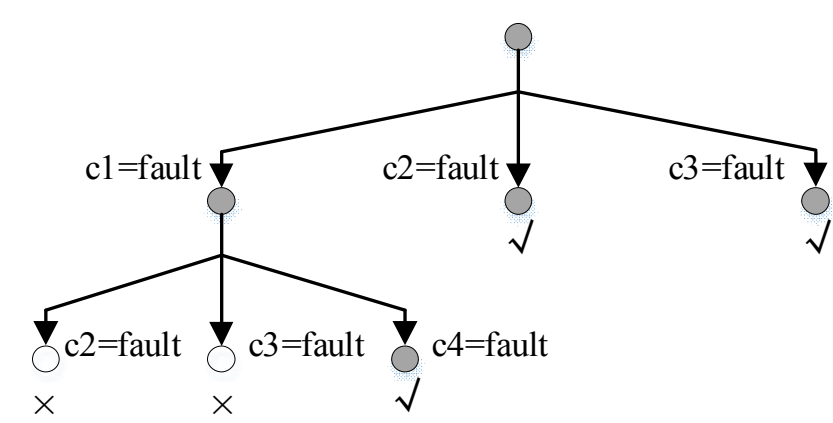

Constituent Kernels

For Conflict 1:

$\{\{\mathrm{c} 1=$ fault $\},\{\mathrm{c} 2=$ fault $\},\{\mathrm{c} 3=$ fault $\}\}$

For Conflict 2:

$\{\{\mathrm{c} 2=$ fault $\},\{\mathrm{c} 3=$ fault $\},\{\mathrm{c} 4=$ fault $\}\}$

Figure 3. A Search Tree to Find Kernels of Conflict 1 and Conflict 2.

\subsubsection{Multiple Diagnoses}

When multiple diagnoses are expected, the diagnosis method is quite different. Figure 4 shows a search tree where blank nodes are candidates and grey nodes will be extended in the following search.

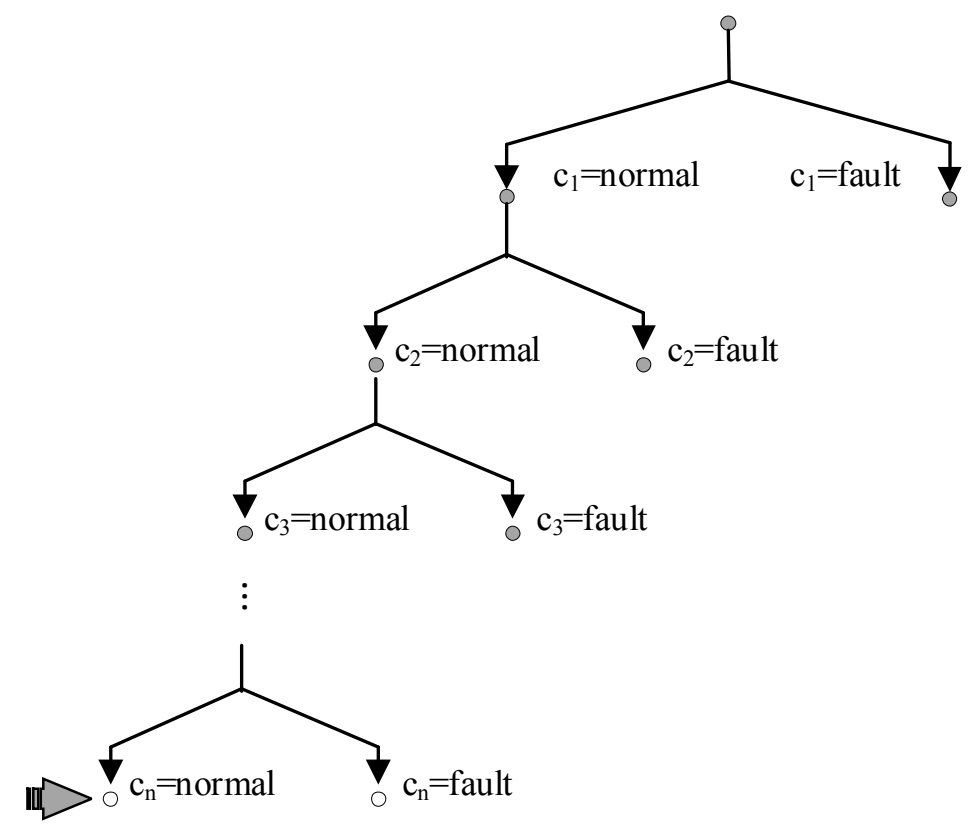

Figure 4. A Search Tree Expanded by Conflict Directed $A^{*}$ Search $\left(\mathrm{CDA}^{*}\right)$ for Multiple Diagnoses.

$\mathrm{CDA}^{*}$ computes the cost of nodes in the search tree according to the prior probability. But if a node contains any conflict, its cost is $\infty$ and the node is skipped. A valid best candidate is found at the leaf node and it is a diagnosis if verified by LTMS. The search-verification iteration ends until enough diagnoses are obtained.

The next section will introduce the shortcomings of current methods and our solutions. 


\section{Conflict and Consistency Directed A* Diagnosis for a Strong-Fault Model}

$\mathrm{CDA}^{*}$ makes full use of conflicts that imply the scope of possible fault components. It is more effective than $\mathrm{BFA}^{*}$ which will not jump over apparently possible candidates but there are still some drawbacks: (1) Although more conflicts can help to find best candidate more accurately, LTMS can only give one conflict one time; (2) Only conflict is utilized to obtain best candidate but consistency between predicted value and observed value implies the possible normal components; (3) The effectiveness of $\mathrm{CDA}^{*}$ is not proven for a strong-fault model.

To overcome the drawbacks above, this paper proposes a Conflict and Consistency Directed $\mathrm{A}^{*}$ diagnosis method illustrated in Figure 5. The Multi-conflict and Multi-consistency LTMS (MCMC-LTMS) checks the consistency of candidate, model and observation and finds as many conflicts and consistencies as possible if it is inconsistent. The $\mathrm{A}^{*}$ search generates the best candidate based on priori probability, conflicts and consistencies. The iteration continues until there are enough diagnoses in diagnosis set.

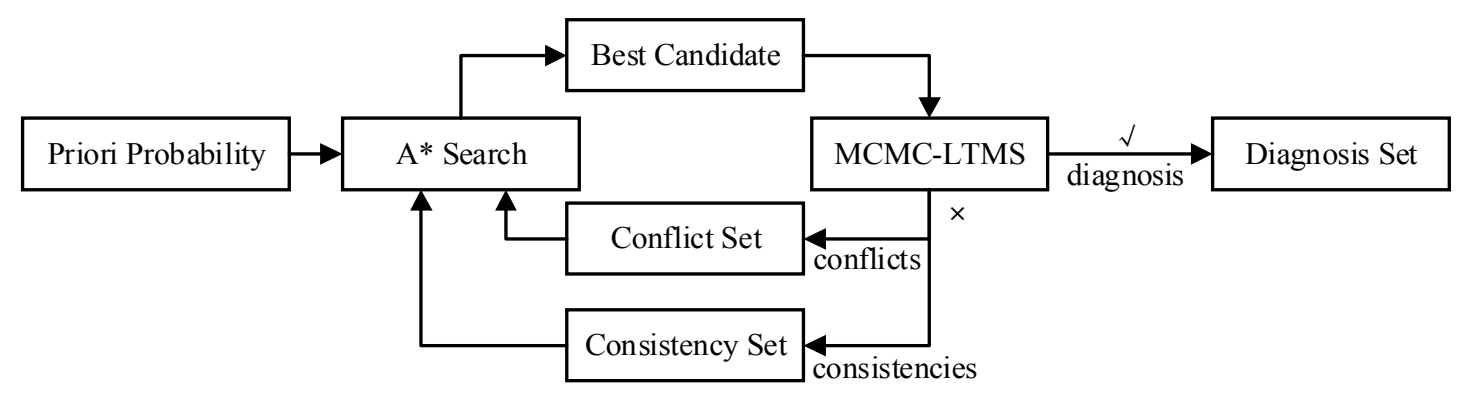

Figure 5. Framework of Conflict and Consistency Directed A* (CCDA).

To accomplish this framework, three problems must be solved. The first one is to represent the model by Boolean variables so that classic reason technique such as unit propagation [30] can be applied. The second problem is MCMC-LTMS, which is quite difficult to achieve because there are insignificant consistencies generated in reasoning. They must be identified and ignored to avoid disturbing the generation of best candidate. How to utilize all the information to generate the best candidate is the last problem. This paper uses the encoding technique in Torta's thesis [29] to address the first one and proposes innovative methods to solve the last two.

\subsection{Model Encoding}

Since there are multi-valued variables (enumeration variables) in a strong-fault model, LTMS cannot reason the system directly. Fortunately, multi-valued variables can be encoded by Boolean variables.

A multi-valued variable $v\left(v=v_{1}, v_{2} \ldots v_{n}\right)$ with $n$ values can be encoded by $n$ Boolean variables where the value (true, false) of each Boolean variable indicates if the value of $v$ is $v_{i}$. Then the assignments in $S D$ are replaced by Boolean variables as shown in (10) where $v$ is a multi-valued variable, $v @ v_{i}$ and $b_{i}$ are Boolean variables.

$$
\begin{aligned}
& \text { multi }- \text { valued variables }: v=v_{1}, v_{2} \ldots v_{n} \\
& \text { boolean variables : v@v } 1, v @ v_{2} \ldots v @ v_{n} \\
& \text { origninal } S D:\left(v=v_{i}\right) \wedge b_{1} \wedge ! b_{2} \\
& \text { encoded } S D: v @ v_{i} \wedge b_{1} \wedge ! b_{2}
\end{aligned}
$$

Besides the replacements in $S D$, additional constraints are inserted to ensure that the assignments of Boolean variables are consistent with Enumeration variables. The first one is MUTEXT constraint, 
as (11), which ensures that any two Boolean variables encoding the same multi-valued variable will not be true at the same time.

$$
\text { MUTEXT :!(v@v } \left.v_{i} \wedge v @ v_{j}\right)
$$

The second one is the COMPLETENESS constraint, as (12), which ensures that there must be at least one Boolean variable encoding multi-valued $v$ is true.

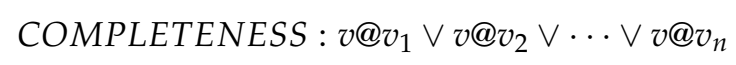

The two constraints make sure that there exists one and only one true variable in any set of Boolean variables encoding an Enumeration variable. By replacing the assignments and adding the extra constraints, original model is converted into a Boolean one that can be solved by LTMS.

\subsection{Multi-Conflict Multi-Consistency LTMS}

LTMS [23] is designed to avoid repeating reasoning. It is composed of node and justification as Figure 6. Nodes can be classified into assumption node, premise node and reason node. In diagnosis, premise nodes are observations and clauses of system model. Justification recording the reasoning process is mainly implemented by unit propagation [31]. All the reasoning rules are illustrated in (13) where $(a)$ is a standard unit propagation which predicts a value of an inner variable/output variable, $(b)$ is a true propagation which means the clause is true and $(c)$ is a false propagation which means the clause is false. The consistency among candidate, model and observation is verified by the three rules.

$$
\begin{aligned}
& \text { (a) } A=\text { false }, A \vee B \Rightarrow B=\text { true } \\
& \text { (b) } A=\text { false } B=\text { true }, A \vee B \Rightarrow \text { true clause } \\
& \text { (c) } A=\text { false } B=\text { false }, A \vee B \Rightarrow \text { false clause }
\end{aligned}
$$

When there is a conflict found in traditional LTMS, the reasoning is stopped. Then, the conflict sets are returned to obtain best candidate.

Although LTMS provides conflict sets to jump over many impossible candidates, it can only get one conflict every time and does not dig the information in system structure deeply. In this subsection, LTMS is improved in two perspectives: multiple conflicts and consistencies.

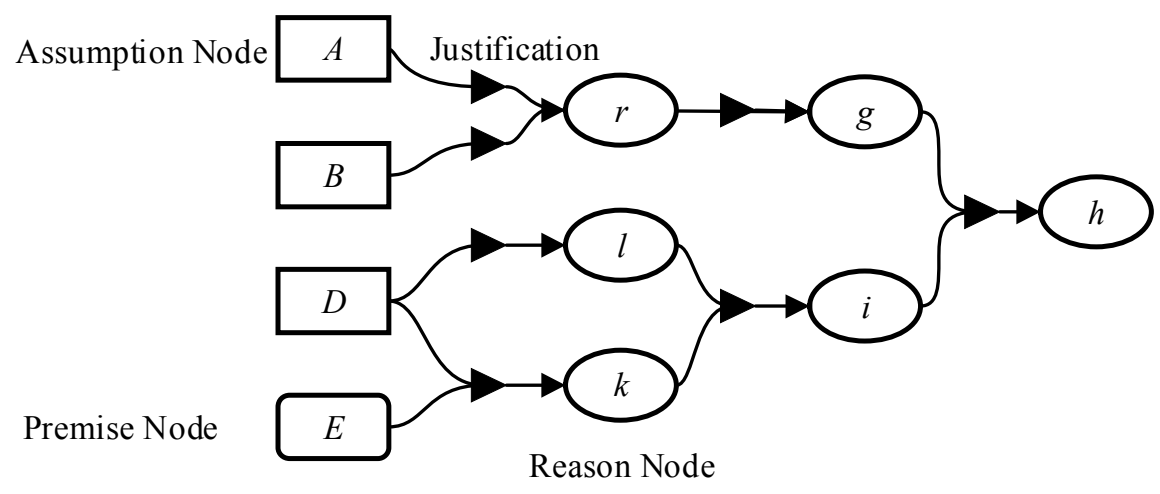

Figure 6. Logic based Truth Maintenance System (LTMS).

\subsubsection{Multi-Conflict LTMS}

More conflicts may help to greatly reduce the range of fault components. For example, in Figure 7 , if the predicted value and observed value of out 2 are different, we can get a conflict set $\{\mathrm{A}=$ normal, $\mathrm{C}=$ normal $\}$ which indicates that there may be fault in component $\mathrm{A}$ or $\mathrm{C}$ but the better candidate can only be determined by priori probabilities. However, if another conflict, for example at out1, is found, it is obvious that component $\mathrm{A}$ is more likely to be faulty. 


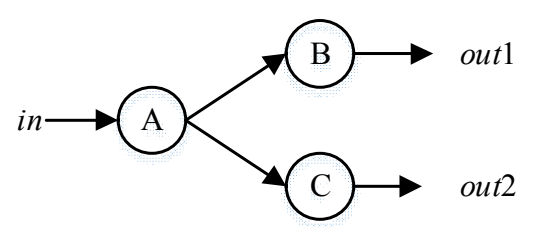

Figure 7. Structure of a Simple System.

To get multiple conflicts in one reasoning process, multi-conflict LTMS does not stop when one conflict is found. To obtain as many conflicts as possible, this paper proposes an out-ignorance in reasoning strategy which means the observed output values do not participate in the reasoning. This technique makes a value can be propagated sequentially even if there is a conflict between predicted and observed value. This helps the reasoning to cover as many components as possible. Conflicts are found in two ways: (1) the differences between predicted values and observed values; (2) false clause as (c) in (13). Conflict sets are obtained by searching back forward until all the assumed modes causing conflicts (or fault clauses) are found.

\subsubsection{Multi-Consistency LTMS}

Conflict reveals the scope of fault components. In the opposite, is there something indicating the range of normal component? Still in Figure 7 , conflict $\{\mathrm{A}=$ normal, $\mathrm{C}=$ normal $\}$ indicates both components $\mathrm{A}$ and $\mathrm{C}$ are possible to be faulty. But if there predicted value and observed value at out 1 is the same, it implies that both component $\mathrm{A}$ and $\mathrm{B}$ are normal. So, component $\mathrm{C}$ is the one probably to be faulty. The example shows the basic idea of consistency, which is formally defined as follows:

A consistency $c$ is an assignment set of mode variables satisfies that (1) there exits an over determined clause set [32] $S D_{c}$ in which any mode variable also exists in $c$; (2) there is no conflicts over $c, S D_{c}$ and observation obs as (14):

$$
c \cup S D_{c} \cup o b s \mid \neq \perp
$$

A consistency $c$ is maximal if there is no super set of $c$ is a consistency. Different from multi-conflict LTMS, multi-consistency finds maximal consistencies.

Consistency is found by true clause but not all true clauses are consistencies. For example, (in ==out) can be converted to $(($ in $\vee$ out $) \wedge($ in $\vee$ !out $))$, where (in $\vee$ !out $)$ is true when in is observed to be true. However, the true clause is insignificant because it does not verify the functionality (in $==$ out). To obtain the significant consistencies, three techniques are adopted: (1) unit propagation delay; (2) unknown ignorance; and (3) false-enumeration ignorance. Unit propagation delay means all clauses can be true or false must be inferred first before conduct unit propagation. Unknown ignorance means if an unknown variable is included in a true clause, the clause is ignored to find consistency. False-enumeration ignorance is the same with unknown ignorance but unknown variable is replaced by false variable encoding an enumeration. Rule 1 makes sure that insignificant clause like (in $\vee$ ! out) can be found. Rule 2 and rule 3 will mark them as invalid clauses for consistency. The consistency can be found by searching back forward from true clauses and "verified" atoms (must be true for variables encoding enumerations and any values for original Boolean variables).

One important thing is that the assignments in a consistency are not ensured to be correct, just like the assignments in a conflict are not always wrong. A Bayesian model is proposed to analyze the relationships among the correctness of assignments, conflicts and consistencies, which is discussed in Section 3.3.1.

\subsubsection{MCMC-LTMS}

This subsection combines the ideas of multiple-conflict and multiple-consistency together. The details of MCMC-LTMS are illustrated by pseudocodes where three routines are the most important: CHECK_CONSISTENCY, MIN_CONFLICT and MAX_CONSISTENCIES. CHECK_CONSISTENCY checks the consistency among system model SD, observation obs and 
candidate $\omega$. MIN_CONFLICT and MAX_CONSISTENCIES find multiple minimal conflicts and multiple maximal consistencies respectively.

Algorithm 1 illuminates the framework of CHECK_CONSISTENCY for MCMC-LTMS. It applies PROPAGATE_FORWARD on clause_set until no propagation is made (flag $==0)$. If there exists a conflict or false clause in any propagation, the route returns false. On the contrast, it returns true.

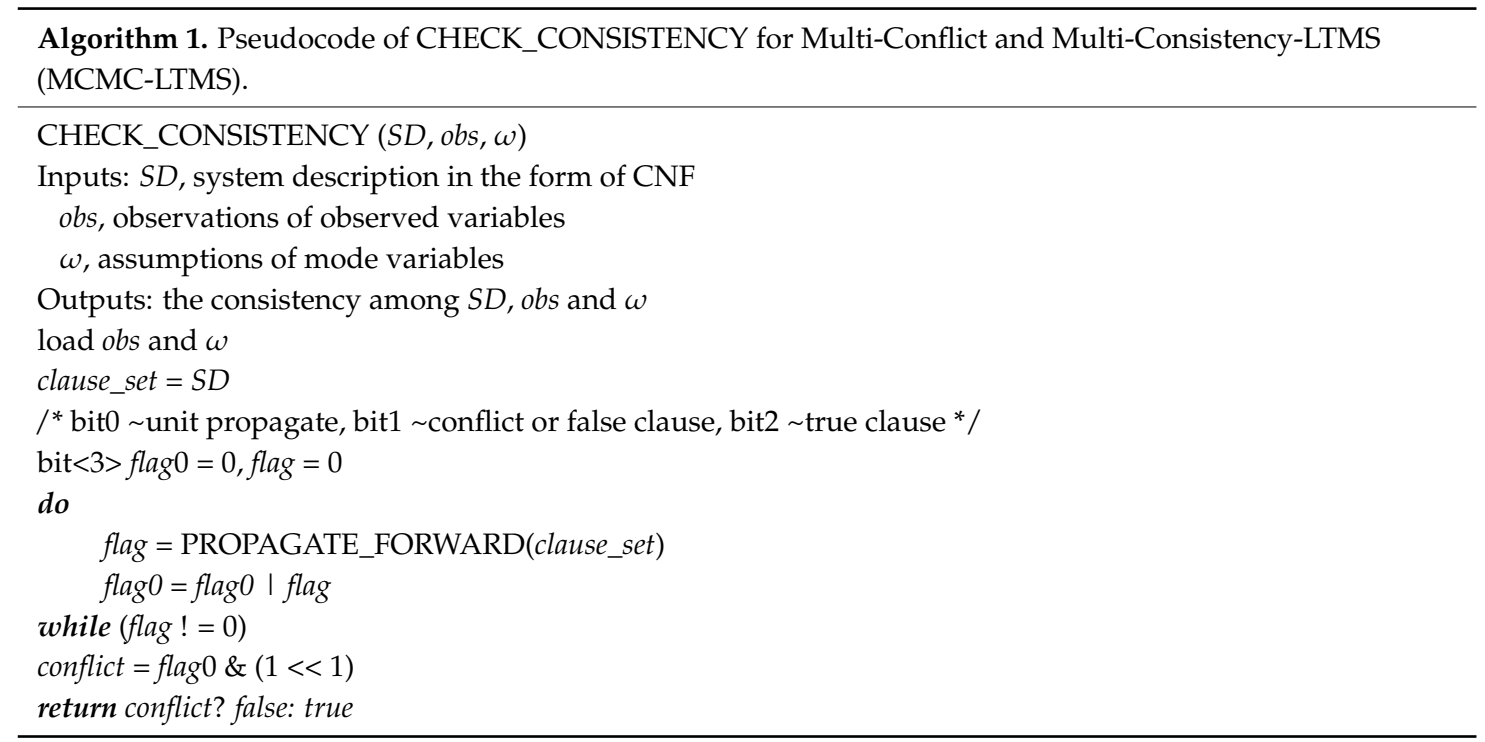

Algorithm 2 shows how to reason by PROPAGATE_FORWARD. For all clauses, CLAUSE_SCAN finds the false, true and unknown literals in clause and stores them in false_assign, true_assign, unknown_literal. When true_assign is not empty, the clause is true. But if the unknown_literal is not empty (rule 2) or there exists false assignment for variable encoding enumeration variables (rule 3), the clause is not valid for consistency. If the unknown_literal are more than one, the clause remains the same. If there is only one unknown literal, unit propagation is applied but delayed (rule 1). If all the literals are false, the clause is false and at least one conflict set can be obtained.

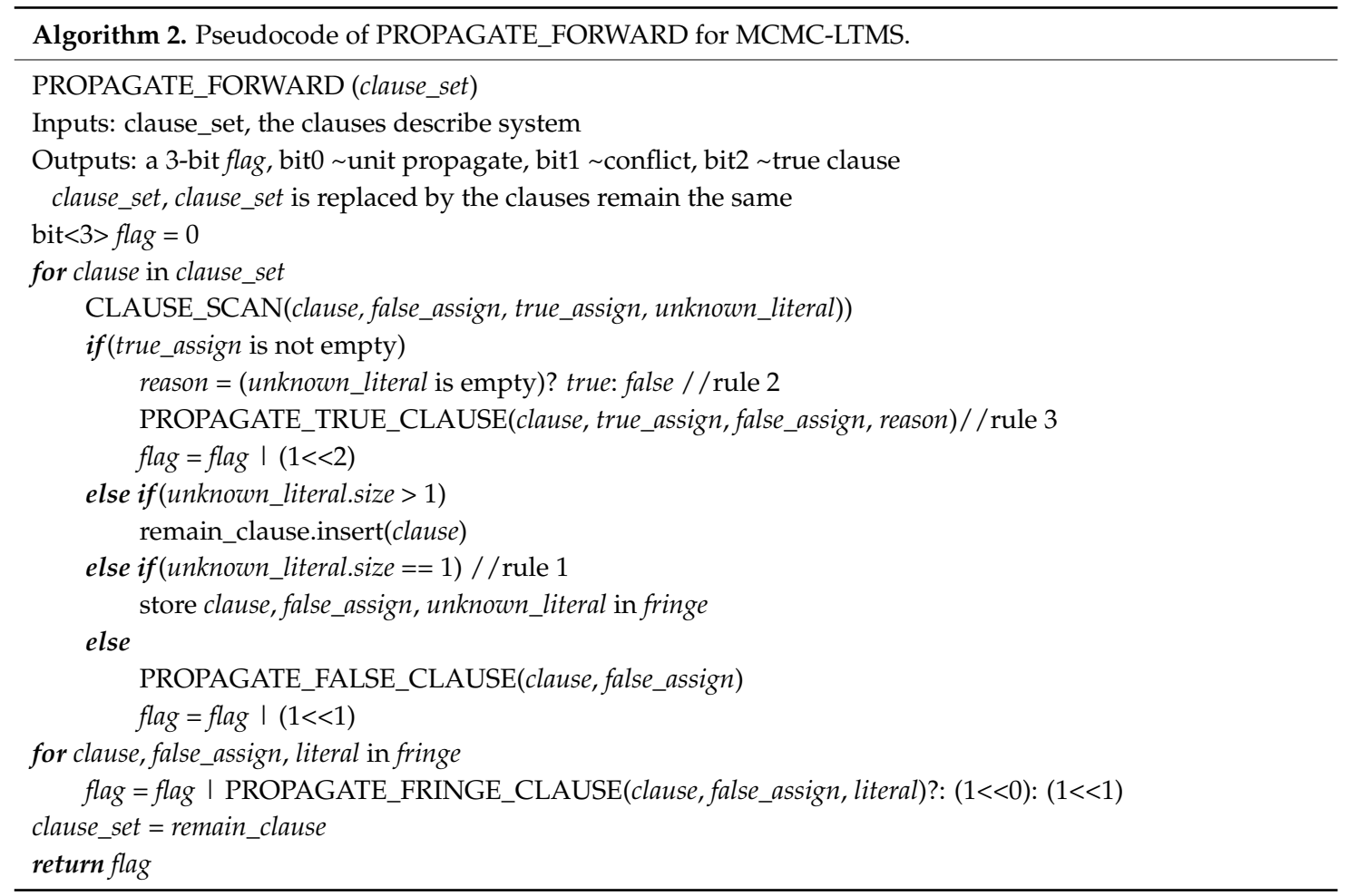


Algorithm 3 illustrates how to obtain multiple minimal conflicts when it is inconsistent. MIN_CONFLICTS searches backward from all the conflict atoms and false clauses, finding conflict sets recursively from down to up. If a conclusion is made by several premises, the results of the premises are combined by MIN_PRODUCT and if a conclusion is made by anyone on several premises, they are combined by MIN_PLUS. Finding the maximal consistencies are similar in Algorithm 4 except that MIN_PRODUCT and MIN_PLUS are replaced by MAX_PRODUCT and MAX_PLUS where PRODUCT means a Cartesian product of sets; PLUS means union set of sets; MIN means the minimal set; and MAX means the maximal set.
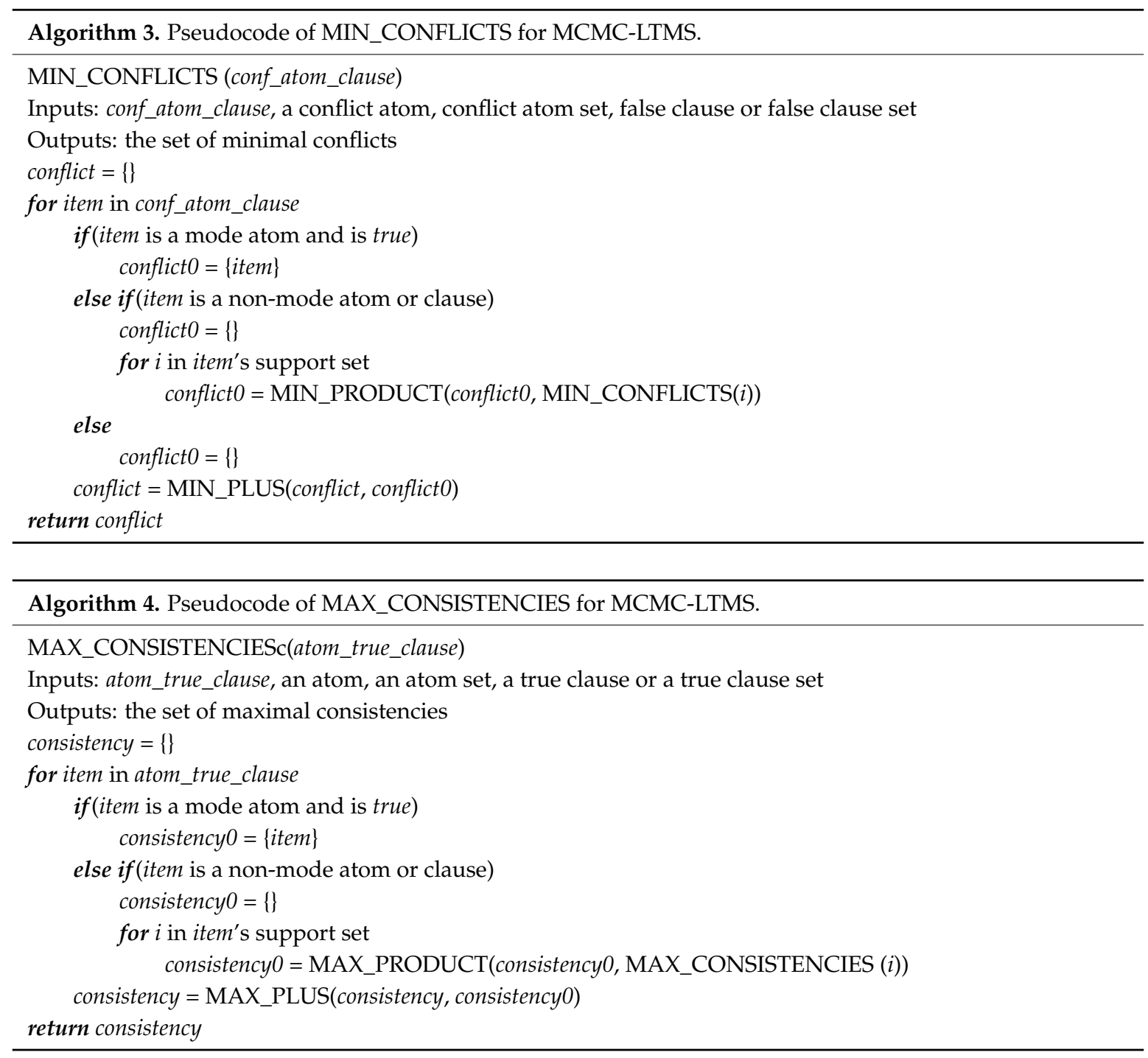

After obtaining the minimal conflicts and consistencies, the $\mathrm{A}^{*}$ search algorithm is employed to generate new candidates.

\section{3. $A^{*}$ Search in Diagnosis}

This subsection describes how to use the $A^{*}$ search to generate the best candidates based on the conflicts and consistencies returned by MCMC-LTMS in two cases: single diagnosis and multiple diagnoses. 
The most important idea of the $\mathrm{A}^{*}$ search is to solve the diagnosis problem as an optimization problem as (15) where $g$ is the evaluation function.

$$
\begin{aligned}
& \max : g(\omega) \\
& \text { subject to }: S D \cup \text { obs } \cup \omega \mid \neq \perp
\end{aligned}
$$

In most cases, unfortunately, the diagnosis optimization problem for a strong-fault model is nonconvex like Figure 8 where the $\mathrm{X}$-axis and $\mathrm{Y}$-axis are modes of component 1 and component 2 respectively and the $Z$-axis is the probabilities of different mode combinations.

$\mathrm{BFA}^{*}$ and $\mathrm{CDA}^{*}$ solve the problem by $\mathrm{A}^{*}$ search algorithm [33] based on mutual, preferential independence (MPI) [22] ensuring that the values of mode variables can be assigned one by one independently. This section discusses how to exploit priori, conflict and consistency in detail.

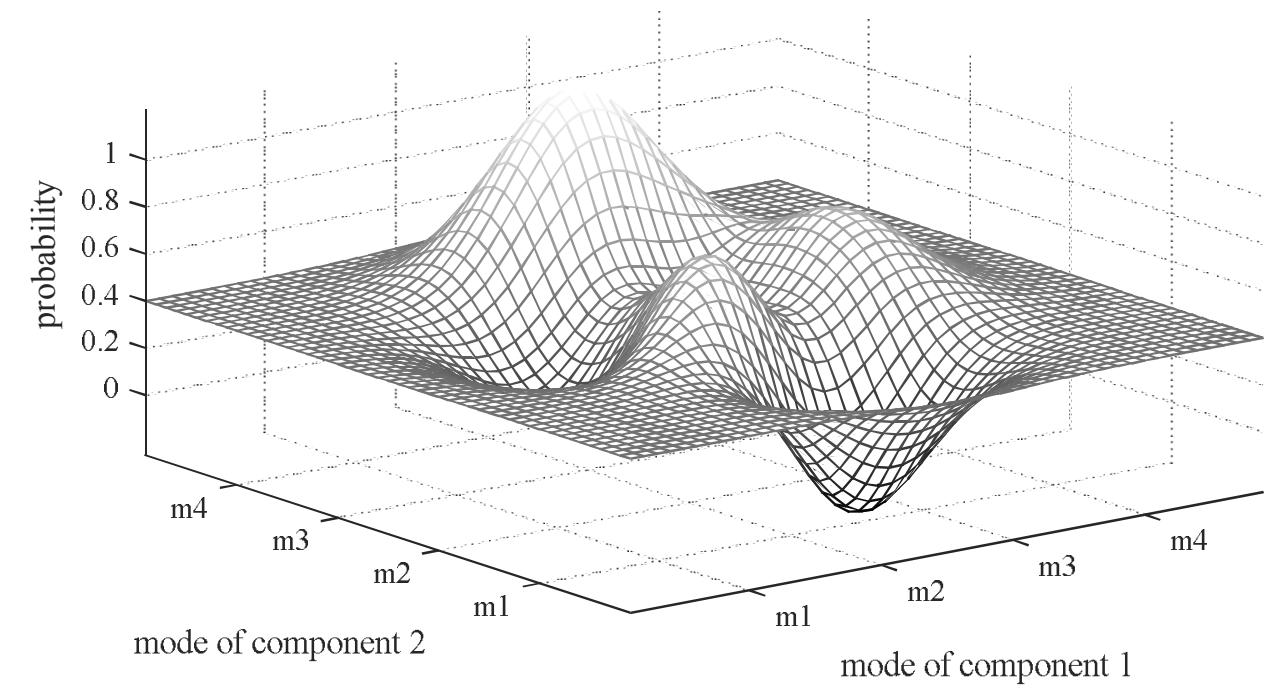

Figure 8. Probabilities of Different Modes Combinations.

\subsubsection{Bayes in Assignments and Possible Conflicts}

Conflict and consistency are different evaluation results of a redundant set. Pulido calls a redundant set a Possible Conflict (PC) [32]. This paper adopts the same terminology but uses PC to represents the unknown result of an equality check between two values (two model prediction values, or one model prediction value and one observation). Traditional methods simply assume that if there is a conflict, the candidate is invalid (false alarm probability is zero); if there is no conflict, the candidate is a diagnosis (missing probability is zero). For the discrete model, the first rule is right according to the definition of consistency-based diagnosis [29]. However, the second rule is not absolutely right for a strong-fault model. One of the most famous examples is "Stuck Fault" [20] which means a component gets stuck on a mode in spite of commands. When the component is in the mode $m$, there will be no conflict even the candidate set the component in "stuck $m$." Although the candidate can be lost because it contains more fault number compared with the candidate assuming the component is in the mode $m$, the oversimplified probability model cannot help us to evaluate the cost if we want to set some mode variables different values from a consistency set. Because if missing alarm is zero, the assignments in a consistency must be right. However, the statement can be wrong. This part analyzes the influence relations between mode variable assignments and possible conflicts using the Bayesian method and proposes a new simplified probability model, assuming that: (1) each mode assignment influences each involved PC independently; (2) false alarm probability is zero (due to the definition of consistency-based diagnosis); and (3) missing alarm probability is a small constant. 
The bipartite graph in Figure 9 illustrates the influence relationships between mode variables and PCs, where $\omega_{i}$ is the assignment for a mode variable and $\mathrm{PC}_{i}$ is a possible conflict. Possible conflict PC $=1$ means the $\mathrm{PC}$ is consistent (consistency) and $\mathrm{PC}=0$ means inconsistent (conflict). $\omega_{i}=1$ means the assignment is correct and $\omega_{i}=0$ means the assignment is wrong. Without confusion, $\omega_{i}$ is also used to represent the assigned mode value of component $i$. In the worst case, any assignment is involved in all possible conflicts as in Figure 9. However, usually one possible conflict only involves several assignments. The $k_{j}$ components involved in the $j$ th possible conflict $\mathrm{PC}_{j}$ are denoted by $\omega_{j 1}, \omega_{j 2} \cdots \omega_{j k_{j}}$. Based on the structure in Figure 9 and results of PCs, the probability of mode assignments is evaluated by (16) to find the best candidate.

$$
\begin{aligned}
P\left(\omega_{1}, \omega_{2} \cdots \omega_{n} \mid \mathrm{PC}_{1}, \mathrm{PC}_{2} \cdots \mathrm{PC}_{m}\right)= & \frac{P\left(\omega_{1}, \omega_{2} \cdots \omega_{n}, \mathrm{PC}_{1}, \mathrm{PC}_{2} \cdots \mathrm{PC}_{m}\right)}{P\left(\mathrm{PC}_{1}, \mathrm{PC}_{2} \cdots \mathrm{PC}_{m}\right)} \\
= & \prod_{i=1}^{n} P\left(\omega_{i}\right) \\
& \times \prod_{j=1}^{m} P\left(\mathrm{PC}_{j} \mid \omega_{j 1}, \omega_{j 2} \cdots \omega_{j k_{j}}\right) \\
& \times \frac{1}{P\left(\mathrm{PC}_{1}, \mathrm{PC}_{2} \cdots \mathrm{PC}_{m}\right)}
\end{aligned}
$$

where $\prod_{i=1}^{n} P\left(\omega_{i}\right)$ is the priori probability of assignments, $\prod_{j=1}^{m} P\left(\mathrm{PC}_{j} \mid \omega_{j 1}, \omega_{j 2} \cdots \omega_{j k_{j}}\right)$ is the conditional probability mentioned in $(7)$ and $P\left(\mathrm{PC}_{1}, \mathrm{PC}_{2} \cdots \mathrm{PC}_{m}\right)$ is the normalization factor. Finding the best candidate means to solve optimization problem (17) where $\vec{\omega}=\left[\omega_{1}, \omega_{2} \cdots \omega_{n}\right]^{T}$.

$$
\max : g(\vec{\omega})=\prod_{i=1}^{n} P\left(\omega_{i}\right) \times \prod_{j=1}^{m} P\left(\mathrm{PC}_{j} \mid \omega_{j 1}, \omega_{j 2} \cdots \omega_{j k_{j}}\right)
$$

It is difficult to solve (17) because the probabilities in this formula are coupled with the real structure of system. To provide a general diagnosis method, this paper assumes that each mode assignment $\omega$ effects the probability independently and the simplified distribution model for $P\left(\mathrm{PC}_{j} \mid \omega_{j 1}, \omega_{j 2} \cdots \omega_{j k_{j}}\right)$ is shown in Table 1 where $\varepsilon$ (missing alarm probability) is positive number close to zero. If a fault may not cause a conflict, $\varepsilon$ should be larger.

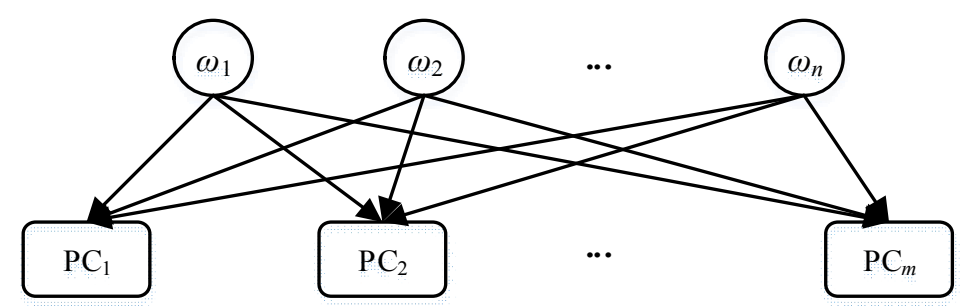

Figure 9. Structure of Mode Variables and Possible Conflicts.

Table 1. Simplified Distribution of Mode Variable Assignments and Possible Conflicts.

\begin{tabular}{ccc}
\hline Mode Assignments & $\mathrm{PC}_{j}$ & Conditional Probability \\
\hline \multirow{2}{*}{$f$ is the number of wrong assignments } & 1 & $\varepsilon^{f}$ \\
\cline { 2 - 3 } & 0 & $1-\varepsilon^{f}$ \\
\hline
\end{tabular}

The distribution in Table 1 indicates that: when all the assignments in a PC is correct $(f=0)$, PC must be consistent $\left(P(P C=1)=\varepsilon^{0}=1\right.$ and $\left.P(P C=0)=1-\varepsilon^{0}=0\right)$; when one assignment is wrong, $\mathrm{PC}$ is probability to be a conflict $(P(\mathrm{PC}=1)=\varepsilon$ and $P(\mathrm{PC}=0)=1-\varepsilon)$; when there are multiple assignments are wrong, $\varepsilon^{f}$ and $1-\varepsilon^{f}$ are the probability of consistency and conflict respectively. Briefly, 
if $\mathrm{PC}_{j}=0$, there must be at least one wrong assignment in $\omega_{j 1}, \omega_{j 2} \cdots \omega_{j k_{j}}$; if $\mathrm{PC}_{j}=1$, the probability of any $\omega=1$ in $\omega_{j 1}, \omega_{j 2} \cdots \omega_{j k_{j}}$ is much higher.

To avoid numerical problem, (17) is converted into a cost-based minimization optimization problem (18) where $f_{j}$ is the number of different assignments between new candidate and consistency or conflict (assuming new candidate is correct, the different assignments in consistency or conflict are wrong assignments), $n$ is the number of components and $m$ is the number of PCs.

$$
\begin{aligned}
\min : c(\vec{\omega}) & =-\ln \left(\prod_{i=1}^{n} P\left(\omega_{i}\right) \times \prod_{j=1}^{m} P\left(P C_{j} \mid \omega_{j 1}, \omega_{j 2} \cdots \omega_{j k_{j}}\right)\right) \\
& =-\sum_{i=1}^{n} \ln P\left(\omega_{i}\right)-\sum_{j=1}^{m} \ln P\left(P C_{j} \mid \omega_{j 1}, \omega_{j 2} \cdots \omega_{j k_{j}}\right) \\
& =\underbrace{\sum_{i=1}^{n}\left(-\ln P\left(\omega_{i}\right)\right)}_{\text {prioricost }}+\underbrace{(-\ln \varepsilon) \sum_{j=1, P C_{j}=1}^{\sum_{\text {conflict-solve cost }}^{m}} f_{j}}_{\text {consistency-break cost }}+\underbrace{\sum_{j}^{m}\left(-\ln \left(1-\varepsilon^{f_{j}}\right)\right)}_{j=1, P C_{j}=0}
\end{aligned}
$$

The cost is composed of three parts: priori cost which is determined by the priori probability, consistency-break cost which is the cost to assign mode values different from the assignments in consistencies and conflict-solve cost which is the similar to break consistency cost but assigns different values from conflicts. Equation (18) indicates that: (1) any different assignment from consistency results in a high punishment in the cost; (2) If there is no different assignment from any conflict, the cost will be positive infinite and the more different assignments from the conflicts, the less the cost is. To minimize the cost function, the consistency-break cost prefers to reserve the assignments in all consistencies and the conflict-solve cost prefers to change all assignments in conflicts. With the same priori probability, different assignments from consistency will increase cost but different assignments from conflict will reduce cost. However, in most cases, the more different assignments from conflict, the priori cost will be larger. Best candidate should make a balance among priori, breaking consistency and solving conflict.

The three parts of cost function in (18) contribute differently to the whole cost. Figure 10 shows the curve of natural logarithm function $y=\ln (x)$ in which $y$ is close to zero when $x$ is close to 1 and $y$ is close to negative infinite when $x$ is close to zero. For priori cost, usually $P\left(\omega_{i}\right)$ for normal mode is close to 1 but close to 0 for fault mode. When finding a candidate containing fault modes, priori cost is not a small quantity. In consistency-break cost, because $\varepsilon$ is a positive number close to zero, $\ln \varepsilon$ is not a small quantity and when assignments in candidate are different from those in consistency, consistency-break cost contributes a lot. And consistency-break cost is zero if the assignments in consistencies keep the same. For conflict-solve cost, when assignments in conflicts are not changed, it is infinite, however it is a small quantity when some assignments in conflicts are changed and conflict- solve cost does not decrease much when $f$ increases.

So, (18) can be approximated by (19).

$$
\operatorname{cost}(\vec{\omega})=\left\{\begin{array}{l}
\begin{array}{c}
\infty, \quad \text { there exists unsolved conflict } \\
\sum_{\text {priori cost }}^{n}\left(-\ln P\left(\omega_{i}\right)\right)
\end{array}+\underbrace{(-\ln \varepsilon) \sum_{j=1, P C_{j}=1}^{m} f_{j}}_{\text {consistency-break cost }}, \text { else }
\end{array}\right.
$$

The only problem for (19) is that, to construct a heuristic function for A* search, consistency-break cost should be in the form of a sum according to component, rather than a sum according to PC. Fortunately, it is very easy to convert. The converted formula is shown in (21). 


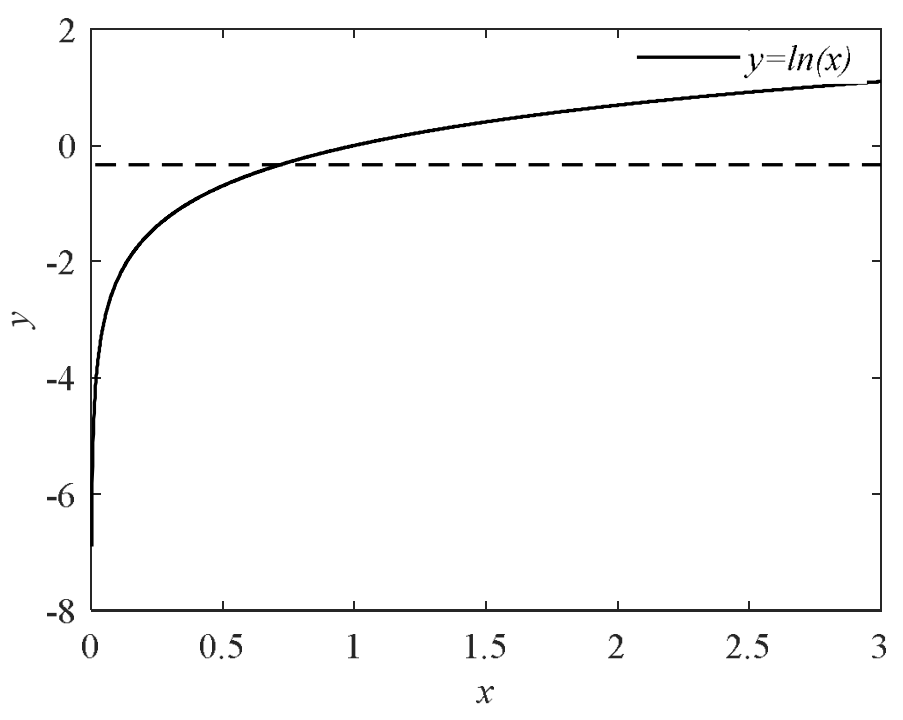

Figure 10. $y=\ln (x)$

\subsubsection{Single Diagnosis-CCDLSA*}

With the MPI property, finding a single diagnosis is relatively simple. The first best candidate $\omega$ is generated by assigning each mode variable the most likely value (for example, all components are normal) according to the prior probability. If there is no conflict found, $\omega$ is a diagnosis and else new candidate must be generated based on the information returned by MCMC-LTMS.

Williams B. C. et al. [22] employs kernel constitutes to find the next best candidate. The core idea behind kernel constitutes is that even if a component is set as fault by mistake, it will not cause more conflicts. However, the diagnosis in solution space for a strong-fault model is nonmonotonic which may cause the expansion of conflict. For example, assuming component B is faulty in Figure 7. If detect a conflict at out 1 (conflict set $\{\mathrm{A}, \mathrm{B}\}$ ) and the kernel constitute algorithm takes $\{\mathrm{A}\}$ as the candidate by mistake. For a weak-fault model, the only mode of A can be fault and the conflict set will still be $\{A, B\}$ because setting component $A$ as fault predicts no wrong value at the output of $A$. However, for a strong-fault model, a specific fault mode must be assigned to component $A$ and a fault mode will cause a wrong output for component $A$ and there will be two conflict sets $\{A, B\}$ and $\{A, C\}$. The expansion of conflict causes "combinatorial explosion" and a strong-fault model cannot adopt kernel constitutes method.

In this subsection, we propose a Conflict and Consistency Directed Local Sequential A* search $\left(\right.$ CCDLSA $\left.^{*}\right)$ to solve this problem where "local" means only components involved in conflicts are searched and "sequential" means new possible fault components are added sequentially. A* algorithm is employed in the search.

$$
<P\left(\omega_{i}\right), f_{i}>
$$

Each node in the search tree is labelled by $(20)$ where $P\left(\omega_{i}\right)$ is the priori probability, $f_{i}$ is the occurrence number of component $i$ in all consistencies in which $\omega_{i}$ is different from the assumption. The cost of the assignments is defined by (21) if all the conflicts are solved.

$$
\sum_{i=1}^{n}\left(-\ln P\left(\omega_{i}\right)+(-\ln \varepsilon) f_{i}\right)
$$

where $n$ is the number of variables in the assignments. If the assignment does not solve all the conflicts, its cost is positive infinite.

Take the system in Figure 7 as an example. Assume each component has three modes. $\left\{\mathrm{A}=\mathrm{m}_{\mathrm{A} .1}, \mathrm{~B}=\mathrm{m}_{\mathrm{A} .1}\right\}$ is a consistency and $\left\{\mathrm{A}=\mathrm{m}_{\mathrm{A} .1}, \mathrm{C}=\mathrm{m}_{\mathrm{C} .1}\right\}$ is a conflict. The search tree is illustrated 
in Figure 11 where the algorithm prefers to believe that $\mathrm{A}^{\prime} \mathrm{s}$ mode is $\mathrm{m}_{\mathrm{A} .1}$ because it does not break any consistency. Algorithms 5 and 6 illustrate the proposed method.

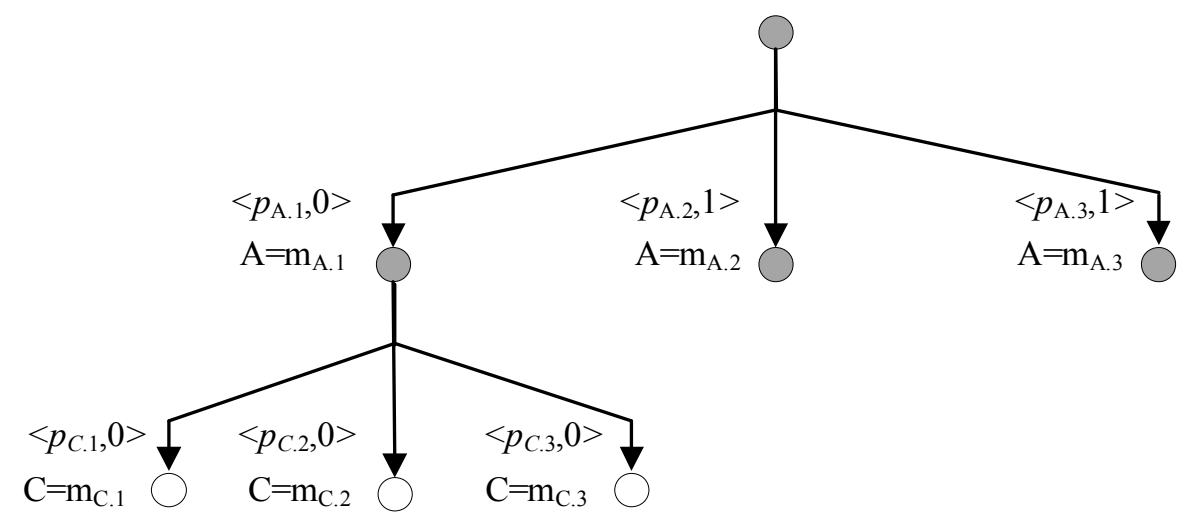

Figure 11. Search Tree of Conflict and Consistency Directed Local Sequential A* search (CCDLSA*)*

Algorithm 5 shows the framework of CCDLSA*. First, all the components are assumed to be normal. If it is not consistent with $S D$ and obs, the minimal conflict and maximal consistency sets are obtained. FAULT_COMPONENT finds possible fault components which are assigned as normal in a conflict set and new possible fault components are pushed back to vector fault_components. $\mathrm{A}^{*}$ BEST_CANDIDATE finds the next best candidate whose consistency is further checked by CHECK_CONSISTENCY. The procedure loops till a consistent diagnosis $\omega$ is found. Because the addition of consistencies will change the cost of existing nodes, only consistencies in the first consistency check are utilized.

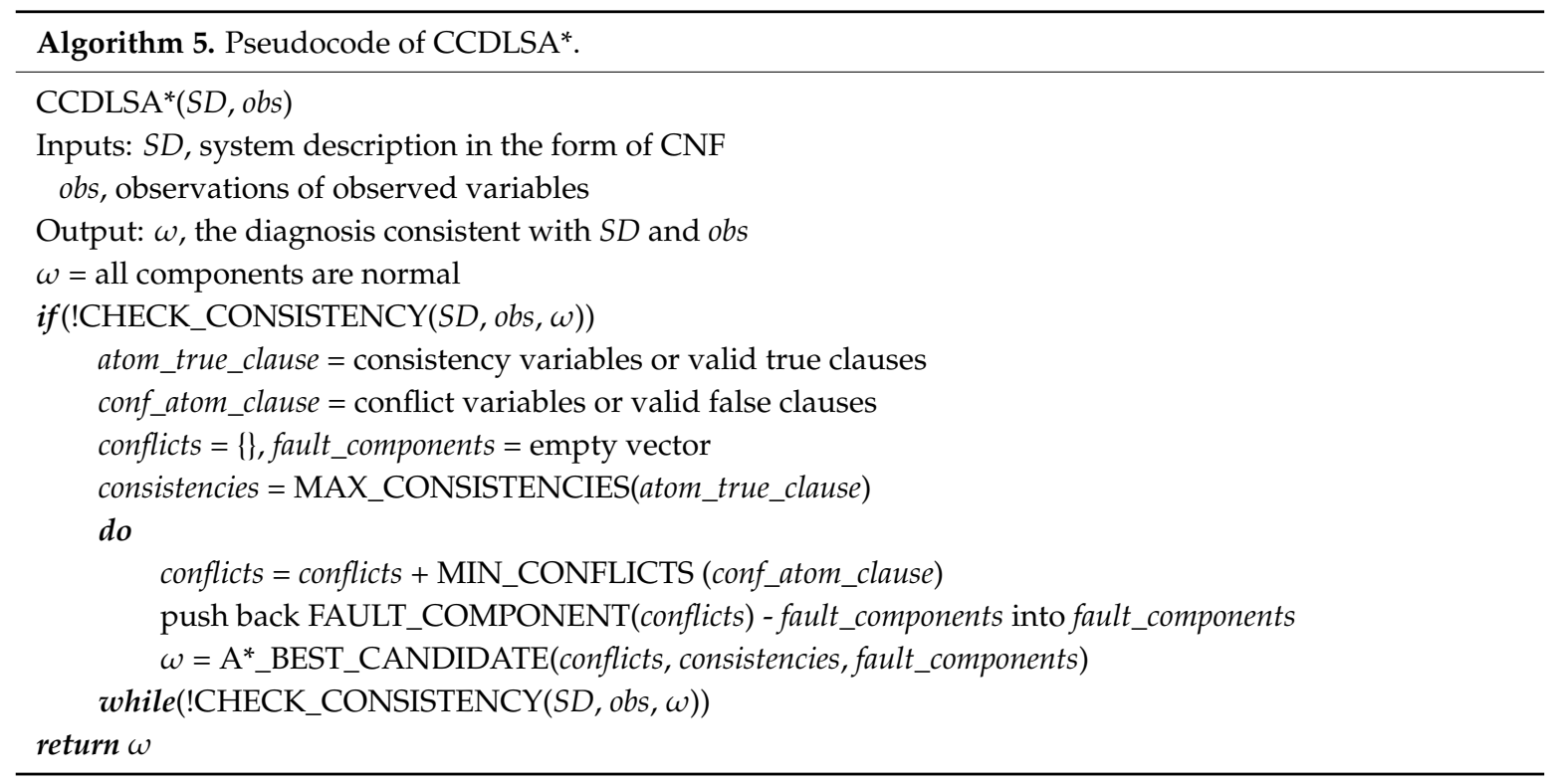

Algorithm 6 shows the kernel algorithm A*_BEST_CANDIDATE. A queue is first initialized by empty set \{\}. Then the node $\omega$ with minimal cost in queue is always popped out. If $\omega$ does not assign values for all components in fault_components, node $\omega$ is expanded by EXPAND where cost is defined by (21). If it does, $\omega$ is the best candidate after adding other default assignments by ADD_DEFAULT.

CCDLSA $^{*}$ is efficient because both conflict and consistency are employed to generate best candidate and the search space is restricted by the possible fault components. However, it is not good at obtaining multiple diagnoses because maybe there exist no enough diagnoses in local space. 


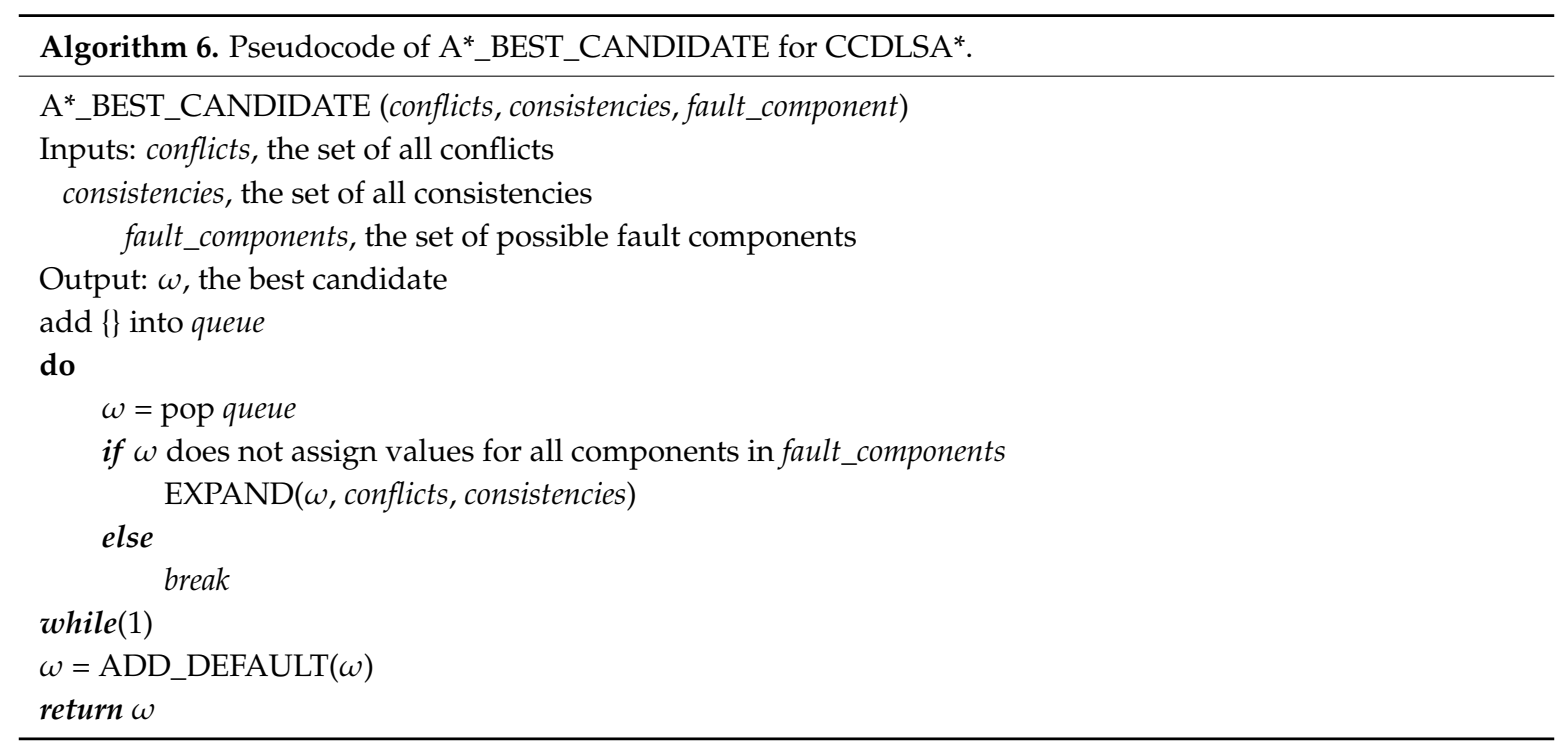

\subsubsection{Multiple Diagnoses-CCDGA*}

The previous subsection proposes a novel focused search diagnosis method to get one diagnosis and this subsection discusses how to get multiple ones. As shown in Figure 8, the search space is nonconvex. It is likely to fall into a local space and fail in getting enough diagnoses. This subsection discusses how to solve this problem by Conflict and Consistency Directed Global A* search (CCDGA*).

Briefly, CCDGA* expands the search space from possible fault components to all the components. Figure 12 illustrates the search tree of a global based diagnosis, for example used in 0 . The basic algorithm shown in Algorithm 7 is similar to the one in Algorithm 5 except that the initial assignments are obtained from the search tree and the loop stops until enough diagnoses are obtained. A*_BEST_CANDIDATE in Algorithm 7 is the same with Algorithm 6 except that fault_components are replaced by all components. CCDGA* can also be used to obtain single diagnosis but CCDLSA* outperforms it because of the less search space.

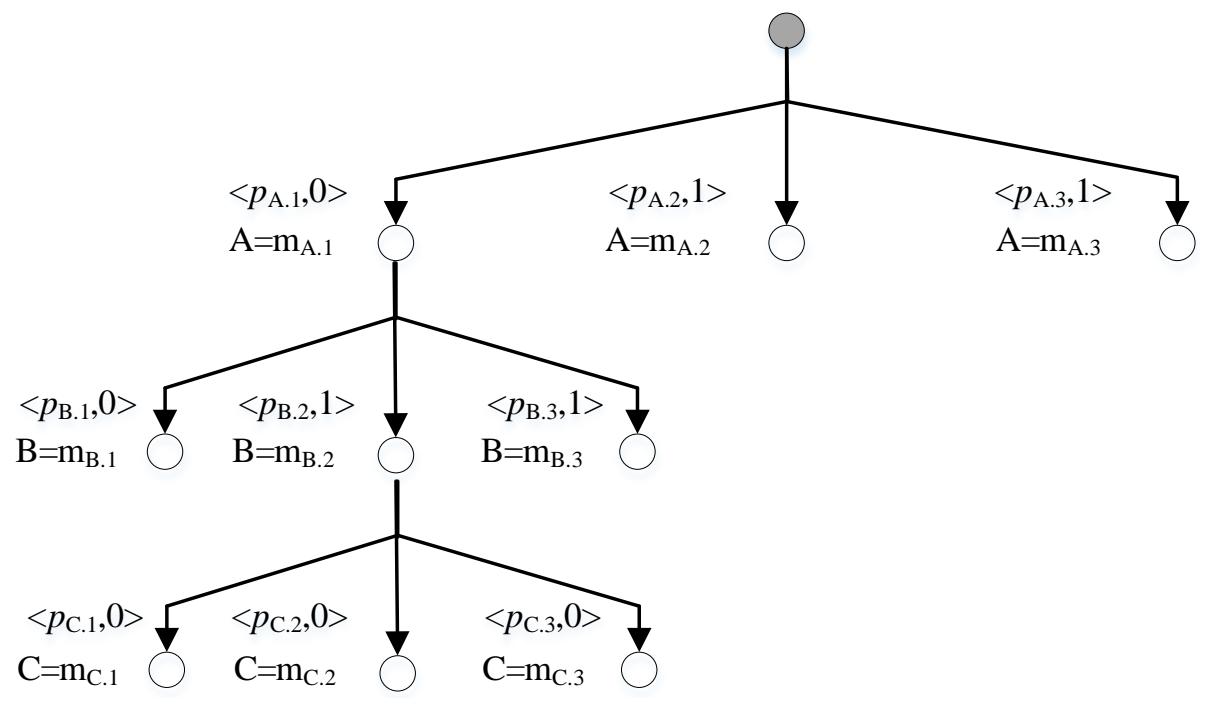

Figure 12. Search Tree of Global based A* Search. 


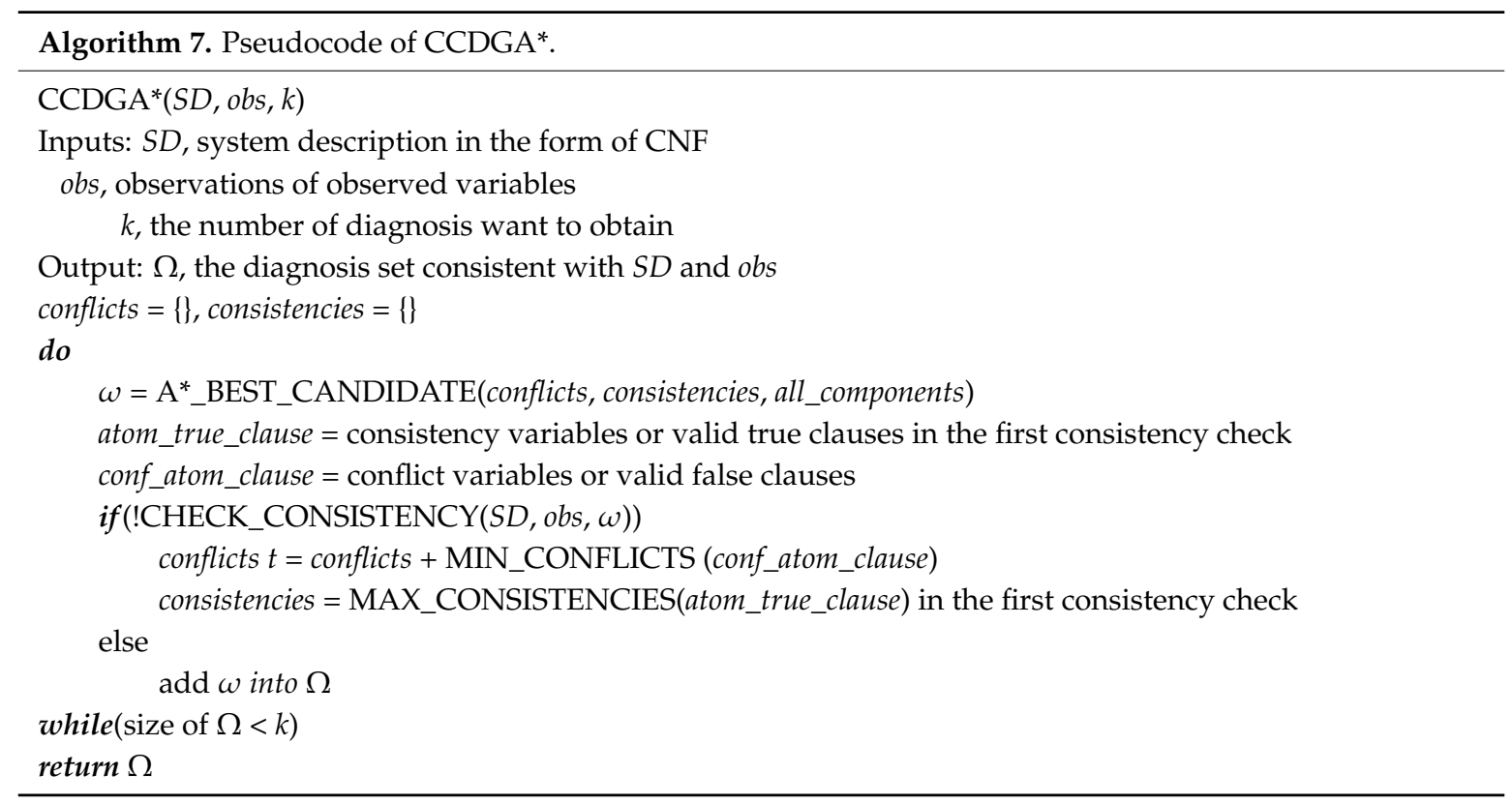

\section{Algorithm Analysis}

This section analyzes methods proposed in Section 3.

\subsection{MCMC-LTMS}

This subsection discusses completeness, coverage and complexity of MCMC-LTMS.

- Completeness

Completeness is defined as the ability to predict all the outputs based on enough inputs. As MCMC-LTMS employs unit propagation as the reason method, it is incomplete in theory. However, "A strong-fault model" makes $S D$ a special subset of propositions. Let's first look at why unit propagation is incomplete. Assuming $A$ and $B$ are two Boolean variables.

$$
\begin{gathered}
(A \vee B) \wedge(! A \vee B) \\
(A \vee ! A) \wedge B
\end{gathered}
$$

Then (22) and (23) are the same proposition in different forms. (22) is a CNF and apparently unit propagation cannot be applied. But (23) tells us that $B$ must be true because $(A \vee ! A)$ must be true. As $\mathrm{CNF}$ is the standard form of unit propagation, unit propagation is incomplete. But the problem no longer exists for a strong-fault model.

To be brief, assume that the behavior of each mode is described by Boolean variables in the form of (24)

$$
m \Rightarrow\left(P_{\text {IN }} \Rightarrow l_{\text {out }}\right)
$$

where $m$ is the mode variable, $P_{I N}$ is a proposition composed of input variables and $l_{\text {out }}$ is the literal of output variable out.

$$
\left(C^{1}{ }_{m, I N} \vee l^{1}{ }_{\text {out }}\right) \wedge\left(C^{2}{ }_{m, I N} \vee l^{2}{ }_{\text {out }}\right) \wedge \cdots \wedge\left(C^{n}{ }_{m, I N} \vee l^{n}{ }_{\text {out }}\right)
$$

Equation (25) is the CNF form of (24) where $C_{m, I N}^{i}$ is a clause composed $m$ and input variables and $l^{i}{ }_{\text {out }}$ is a literal of out. $m$ will be assumed in diagnosis. Inputs are observed or the outputs of other components. If the all the inputs of this component are observed, $\left(C^{i}{ }_{m, I N} \vee l^{i}{ }_{\text {out }}\right)$ is a true /false clause or the value of out can be inferred because the value of $C_{m, I N}^{i}$ is determined. If some inputs of this 
component are not observed, they are sure to be inferred from other components. So, the value of all unknown non-mode variables will be inferred by unit propagation.

As a conclusion, MCMC-LTMS is complete for a strong-fault model.

- Coverage

The coverage discusses if MCMC-LTMS is able to find conflicts covering all fault components. Before analysis, an assumption is made: fault components will surely cause at least one conflict. The assumption means a fault mode will always predict a different output from the normal mode and the outputs of multiple faults will never be counteracted. Although sometimes the assumption is not satisfied, it is necessary to analyze the coverage of MCMC-LTMS theoretically.

When a system is abnormal, there are two methods to find conflict: difference between predicted value and observed value of output and false clause. For the first case, all the outputs are predicted and all the functionalities of the system are verified, which ensures MCMC-LTMS cover all possible fault components, For the second case, the false clause will not stop the propagation of false predicted values. More components may be involved but the real fault component will never be missed.

Under the assumption, MCMC-LTMS can cover all possible fault components. If the assumption is not satisfied, the coverage is not ensured.

\section{- Complexity}

The operation of MCMC-LTMS is composed of two steps: (1) reasoning to get the values of unknown variables and (2) backtracking to find all the conflicts and consistencies.

In the first step, because our object systems are described by a strong-fault model, all the unknown variables can be inferenced by unit propagation and the complexity is $O(n a)$ where $n$ is the number of clauses in a CNF model and $a$ is the time to execute one unit-propagation.

In the second step, if $m$ conflicts and consistencies are found in the whole and $b$ is the time to find one conflict or consistency. The time complexity is $O(m b)$.

As a conclusion, the complexity is $O(n a+m b)$. As a contrast, the complexity of standard LTMS is $\mathrm{O}(n a+m b)$ where $\mathrm{m}$ is 0 or 1 because it only finds the support set of one conflict. Although MCMC-LTMS seems less efficient, it has the same complexity when there is no conflict and MCMC-LTMS saves many reasoning steps when there exist conflicts because it merges information from multiple conflicts.

\section{2. $A^{*}$ Search in Diagnosis}

\subsubsection{Single Diagnosis-CCDLSA*}

\section{- Correctness}

We define the correctness of proposed algorithm as the ability to detect conflict if there exists one and the ability to find a consistent diagnosis. Sometimes a fault will not cause conflict but the problem can only be solved by improving model rather than algorithm.

For a strong-fault model, MCMC-LTMS is complete. It is able to find at least one conflict if exist. Although MCMC-LTMS cannot cover all possible fault components in the first consistency check when the assumption is not satisfied, the any possible fault components missed in the first check will be added to the search space in the next loops. CCDLSA* enumerates all the possible combinations and the real assignments must be in them. So CCDLSA* is sure to terminate and get the diagnosis.

- Complexity

The space and time complexity of CCDLSA* is the same and is related to the number of possible combinations. In the worst case, CCDLSA* must enumerate all the possible combinations which indicates that the complexity is $O\left(m^{n}\right)$ where $m$ is the average assignment number and $n$ is the fault component number. And if consistency eliminates normal components from fault components correctly, the complexity is reduced. 
- Optimality

The $\mathrm{A}^{*}$ search is optimal when the estimated cost of unassigned mode variables in heuristic function is not overestimated. The heuristic function used in this paper set the estimated cost as zero which can never be over the real cost. Therefore, CCDLSA* is optimal.

\section{- Robustness}

To be optimal, the $A^{*}$ search usually conducts multiple backtracking when it finds that the current node is not optimal. If the priori distribution of mode variable is sharp, which means that the probabilities of different values for one mode variable vary a lot, it is easy to distinct different combinations by priori probabilities and less backtracking occurs. If the prior distribution is flat, the priori probabilities of different are similar to each other and more backtracking occurs.

In a summary, if the priori distributions of mode variables are sharp, the CCDLSA* is robust. However, if the priori distributions are flat, the robustness is not ensured.

\subsubsection{Multiple Diagnoses-CCDGA*}

- Correctness

The correctness of CCDGA* is similar to CCDLSA*. Because it traverses the solution space for all the components, it is sure to find all the possible diagnoses.

But if our expected diagnoses number exceeds the existing ones, CCDGA* will traverse the whole solution space and usually not terminate in acceptable time.

- Complexity

The complexity of CCDGA* is also similar to CCDLSA* except that $n$ is the number of all components.

- Optimality

Due to the same analysis for CCDLSA*, CCDGA* is optimal.

- Robustness

When there exit enough diagnoses, robustness of CCDGA* is the same with CCDLSA*: if the priori distributions of mode variables are sharp, the CCDGA* is robust. However, if the priori distributions are flat, the robustness is not ensured. However, if there are fewer diagnoses than the expected number,

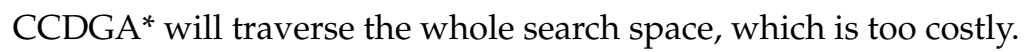

\section{Case Study}

\subsection{Model Introduction}

Figure 13 shows a heat control system composed of battery, switch, heater, sensor and ammeter in satellite. The battery offers power so that the heater can keep other equipment warm and operating well. There is a main switch $S$ to control all the heaters and four switches $s_{i}(i=1,2,3,4)$ to control four heaters. Heater $h_{i}(i=1,2,3,4)$ is composed of two resistors $h_{i 1}$ and $h_{i 2}$. Four sensors $\operatorname{Sen}_{i}(i=1,2,3,4)$ are used to monitor the heating efficiency. Two ammeters $A 1$ and $A 2$ monitor the current at two points.

The modes of all the components are shown in Figure 14 in which normal modes can transfer to both normal modes and fault modes but fault modes can never transfer to normal modes. Table 2 illustrates the behaviors of each component in different modes in details. 


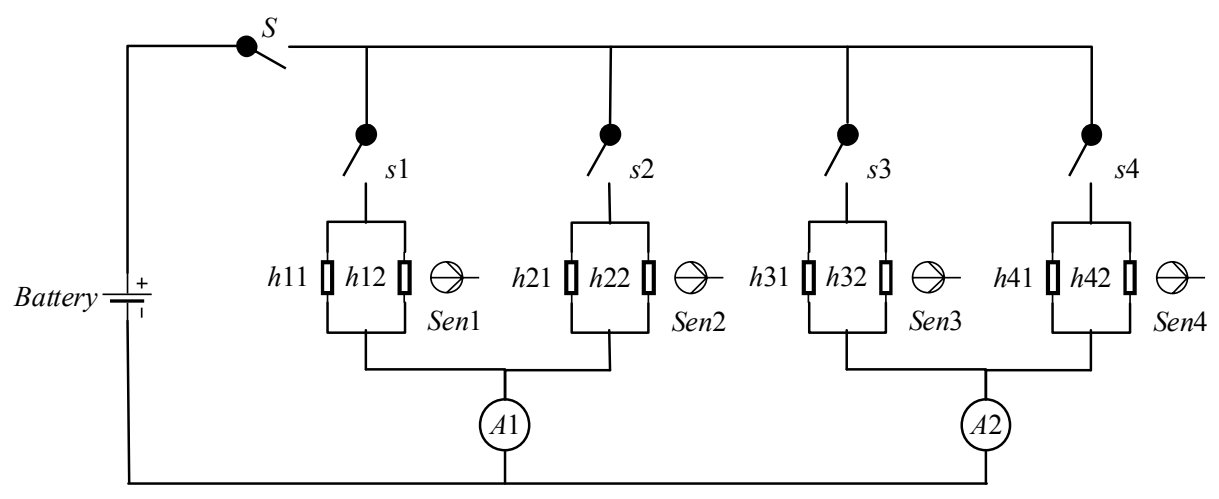

Figure 13. Heat Control System.

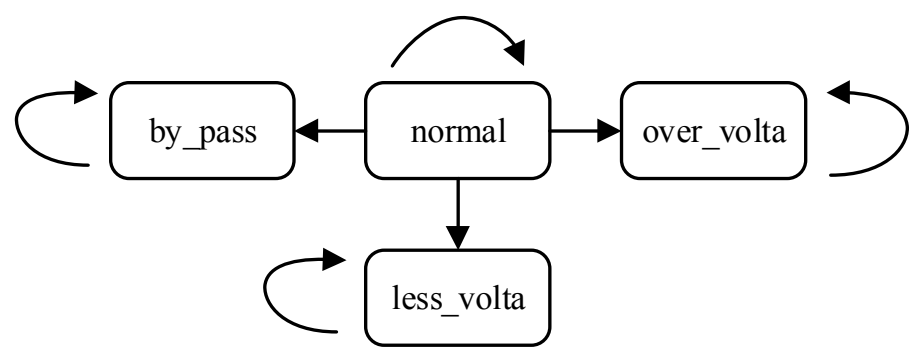

a) mode transition of battery

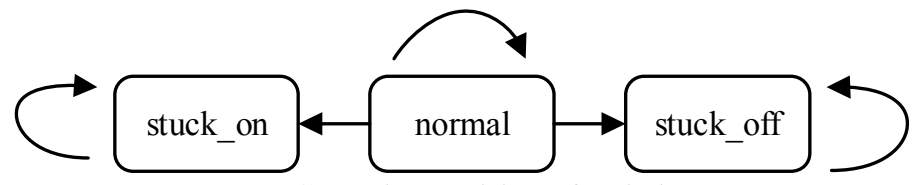

b) mode transition of switch

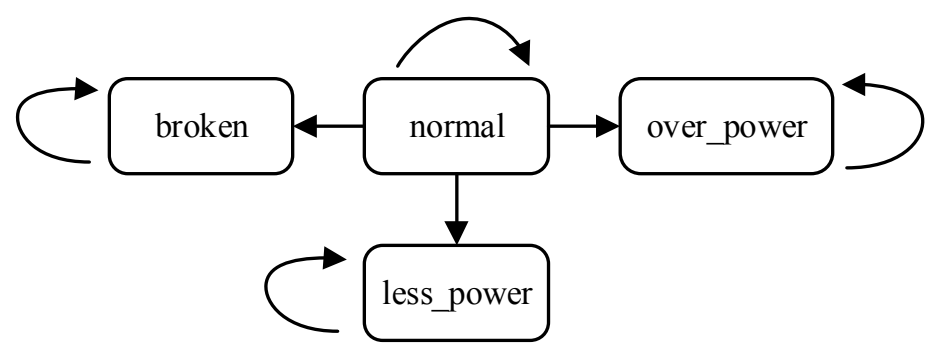

c) mode transition for resistor

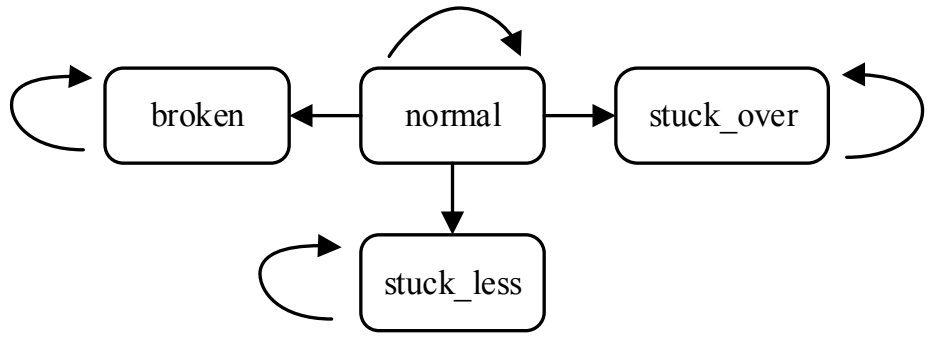

d) mode transition for sensor

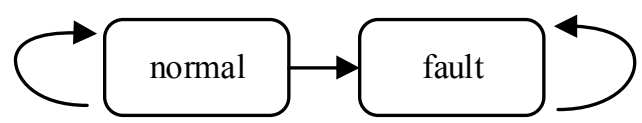

e) mode transition for ammeter

Figure 14. Mode Transitions. 
Table 2. Components, Modes and Behaviors.

\begin{tabular}{|c|c|c|}
\hline Component & Mode & Behavior \\
\hline \multirow{4}{*}{ battery } & normal & The output voltage is normal. \\
\hline & by_pass & The output voltage is zero. \\
\hline & less_volta & The output voltage is lower than normal. \\
\hline & over_volta & The output voltage is higher than normal. \\
\hline \multirow{3}{*}{ switch } & normal & When the switch is on, output is equal to the input. Zero when off. \\
\hline & stuck_on & The switch keeps on ignoring the command. \\
\hline & stuck_off & The switch keeps off ignoring the command. \\
\hline \multirow{4}{*}{ resistor } & normal & $\begin{array}{l}\text { If the input voltage is zero, there is no heat and output current is zero. } \\
\text { If the input voltage is less, power is less and output current exists. } \\
\text { If the input voltage is normal, power is normal and output current exists. } \\
\text { If the input voltage is over, power is over and output current exists. }\end{array}$ \\
\hline & less_power & $\begin{array}{l}\text { If the input voltage is zero, there is no heat and output current is zero. } \\
\text { Otherwise, power is less and output current exists. }\end{array}$ \\
\hline & over_power & $\begin{array}{l}\text { If the input voltage is zero, no heat and output current is zero. } \\
\text { Otherwise, power is less and output current exists. }\end{array}$ \\
\hline & broken & There is no heat and output current is zero. \\
\hline \multirow[t]{4}{*}{ sensor } & normal & $\begin{array}{l}\text { If both resistors generate no heat, output temperature is none. } \\
\text { If both resistors generate heat normally or one is less power but the other is } \\
\text { over power, output temperature is normal. } \\
\text { If one resistor generates heat over power and the other one is normal or over } \\
\text { power, output temperature is over. } \\
\text { In other cases, output temperature is less. }\end{array}$ \\
\hline & stuck_less & Output temperature is less. \\
\hline & stuck_over & Output temperature is over. \\
\hline & broken & Output temperature is broken. \\
\hline \multirow{2}{*}{ ammeter } & normal & The output is the number of input currents. \\
\hline & fault & The output is always zero. \\
\hline
\end{tabular}

The system in Figure 14 is composed of 20 components where there are 4 sensors, 5 switches, 8 resistors, 2 ammeters and 1 battery in whole. The search space is $4 \times 3^{5} \times 4^{8} \times 4^{4} \times 2^{2}=65,229,815,808$ $\approx 6.5 \times 10^{10}$ which is too huge to search by traversing. After encoding, the system is described by Boolean variables and there are 1059 constraint clauses (model) over the system which are also a huge challenge to MCMC-LTMS. The system is employed to demonstrate the proposed methods in the next two subsections where all the experiments are conducted on a i7-8550u, 8G RAM laptop.

\subsection{MCMC-LTMS}

This subsection demonstrates the ability of MCMC-LTMS to find conflict and consistency. The 11 observations are shown in Table 3 where $\mathrm{CS}$ is the command for switch S, cs1 cs4 are the commands for s1 s4, t1 t4 are the output values of Sen1 Sen 4 and $\mathrm{c} 1$ and $\mathrm{c} 2$ are the monitored currents of $\mathrm{A} 1$ and $\mathrm{A} 2$.

Table 3. Observations for Heat Control System.

\begin{tabular}{cccccccccccc}
\hline Variable & cS & cs1 & cs2 & cs3 & cs4 & t1 & t2 & t3 & t4 & c1 & c2 \\
\hline Value & true & true & true & true & true & norm & norm & less & norm & four & four \\
\hline
\end{tabular}

Table 4 shows the conflicts and consistencies found by MCMC-LTMS where there are 1 conflict and 5 consistencies. Based on the structure in Figure 13, it is obvious that the conflict locates the fault 
component in sensor 3 and its upstream components. The first three consistencies mean that branches 1,2 and 4 including three sensors are normal. The last two consistencies extend the normal scope to h31, h32, A1 and A2. The most interesting consistency is \{battery, S, s3, s4, h31, h32, h41, h42, A2\} in which h31 and h32 may be in fault mode less_power in theory but because the predicted outputs at A1 and A2 are consistent with observed values, h31 and h32 are included in consistency.

Table 4. Conflict and Consistency Found by MCMC-LTMS.

\begin{tabular}{ll}
\hline Type & Set \\
\hline Conflict & $\{$ battery, S, s3, h31, h32, sen3\} \\
\hline & $\{$ battery, S, s1, h11, h12, sen1\} \\
& $\{$ battery, S, s2, h21, h22, sen2\} \\
Consistency & $\{$ battery, S, s4, h41, h42, sen4\} \\
& $\{$ battery, S, s1, s2, h11, h12, h21, h22, A1 \\
& $\{$ battery, S, s3, s4, h31, h32,h41, h42, A2 $\}$ \\
\hline
\end{tabular}

The result in Table 4 indicates that MCMC-LTMS can effectively analyze system behaviors and find conflict and consistency set based on the structure information hidden in encoded model.

\section{3. $A^{*}$ Search in Diagnosis}

\subsubsection{Single Diagnosis}

Best First $A^{*}$ search $\left(\mathrm{BFA}^{*}\right)$, Conflict Directed $\mathrm{A}^{*}$ search $\left(\mathrm{CDA}^{*}\right.$, multiple fault version, single fault version cost too much time), $\operatorname{CCDLSA}^{*}(\varepsilon=1.0 \mathrm{e}-2)$ and $\operatorname{CCDGA}^{*}(\varepsilon=1.0 \mathrm{e}-2)$ were evaluated to find one diagnosis for the same scenario in Table 3.

Figure 15 provides the diagnosis process of CCDLSA* where the algorithm tried 3 candidates in all and the diagnosis results showed that sensor Sen3 was in stuck_less fault mode and other components were normal. The correctness of the diagnosis can be easily verified according to system structure and component behavior. To be brief, the diagnosis processes of other algorithms are not given in this paper. All the left 3 algorithms had the same diagnosis results but different diagnosis time and tried candidates number.

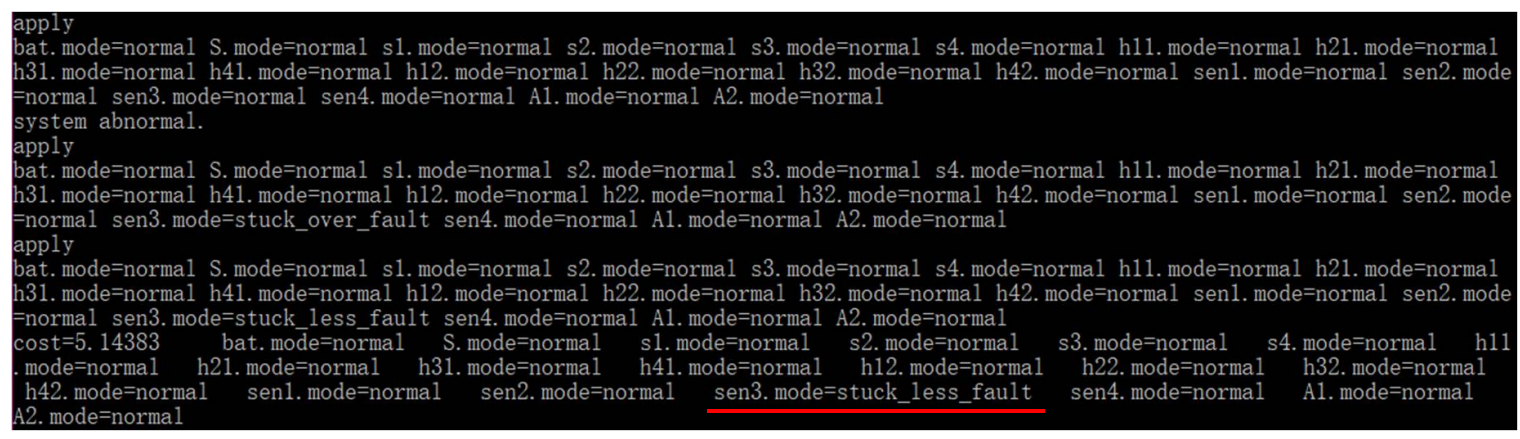

Figure 15. Diagnosis Process of CCDLSA* for Single Diagnosis.

To compare the performances of different methods, BFA* is adopted as the criterion. The "Ratio," defined by (26), in the next 4 tables are the percentages of "algorithm" based on BFA* for different "value"s.

$$
\text { Ratio }(\text { algorithm })=\frac{\text { value of algorithm }}{\text { value of } B F A *} \times 100 \%
$$

The time for 10 experiments is shown in Table 5 where "Ave" means the average time and "Ratio" means the percentages of diagnosis time based on BFA*. We can see that the time for 10 experiments 
fluctuates slightly around the average time which is also shown in Figure 16. The small fluctuation implies that all of the 4 algorithms are stable and proves the robustness of the proposed methods. In average, CCDLSA* and CCDGA* cost less time than BFA* and CDA*. Due to the smaller search space of CCDLSA*, it was slightly better than CCDGA*

Table 5. Single Diagnosis Time(s) for BFA*, CDA*, CCDLSA* and CCDGA*.

\begin{tabular}{ccccccccccccc}
\hline Algorithm & $\mathbf{1}$ & $\mathbf{2}$ & $\mathbf{3}$ & $\mathbf{4}$ & $\mathbf{5}$ & $\mathbf{6}$ & $\mathbf{7}$ & $\mathbf{8}$ & $\mathbf{9}$ & $\mathbf{1 0}$ & Ave & Ratio \\
\hline BFA $^{*}$ & 1.787 & 1.711 & 1.620 & 1.712 & 1.738 & 1.883 & 1.702 & 1.782 & 1.738 & 1.680 & 1.74 & $100.00 \%$ \\
CDA $^{*}$ & 1.644 & 1.756 & 1.711 & 1.668 & 1.676 & 1.640 & 1.687 & 1.650 & 1.682 & 1.64 & 1.68 & $96.55 \%$ \\
CCDLSA $^{*}$ & 1.165 & 1.163 & 1.156 & 1.169 & 1.131 & 1.19 & 1.134 & 1.144 & 1.147 & 1.153 & 1.16 & $66.67 \%$ \\
CCDGA $^{*}$ & 1.191 & 1.24 & 1.252 & 1.311 & 1.183 & 1.183 & 1.178 & 1.256 & 1.267 & 1.319 & 1.24 & $71.26 \%$ \\
\hline
\end{tabular}

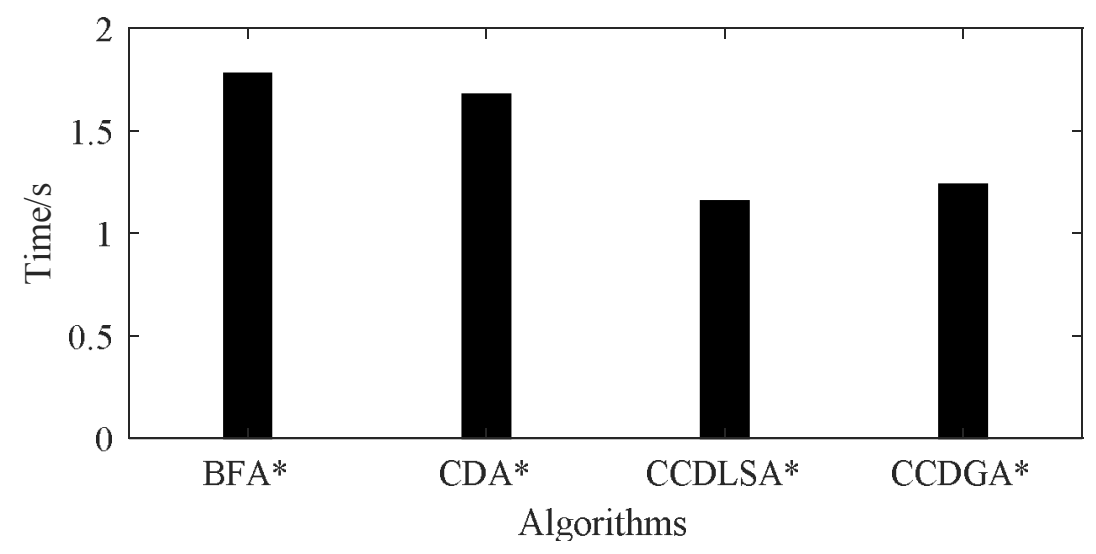

Figure 16. Average Diagnosis Time for BFA*, $\mathrm{CDA}^{*}, \mathrm{CCDLSA}^{*}$ and CCDGA*.

The numbers of nodes for search are shown in Table 6 where "Tried Candidates" means the number of candidates tried by the algorithm, "Expanded Nodes" means the number of nodes expanded in the search tree and "Node in Queue" means the number of the leaf nodes in the search tree when the diagnosis is finished. "Num" means the number of nodes and the percentages "Ratio" beside the numbers are the ratios of their node numbers and the node number for BFA*. Although CDA* tried fewer candidates than $\mathrm{BFA}^{*}$, the numbers of nodes expanded and in queue were still much larger than CCDLSA $^{*}$ and CCDGA* CCDLSA* tried the least candidates, expanded least nodes and left least nodes in the queue as well.

Based on the analysis about Tables 5 and 6 and Figure 16, we can see that CCDLSA* and CCDGA* can avoid invalid node expansion effectively. This feature helps the two proposed algorithms consume less memory space and time to diagnose.

Table 6. Tried Candidates, Expanded Nodes and Nodes in Queue for Single Diagnosis.

\begin{tabular}{ccccccccc}
\hline \multirow{2}{*}{ Algorithm } & \multicolumn{2}{c}{ BFA* $^{*}$} & \multicolumn{2}{c}{ CDA $^{*}$} & \multicolumn{2}{c}{ CCDLSA $^{*}$} & \multicolumn{2}{c}{ CCDGA $^{*}$} \\
\cline { 2 - 8 } & Num & Ratio & Num & Ratio & Num & Ratio & Num & Ratio \\
\hline Tried Candidates & 11 & $100.00 \%$ & 8 & $72.72 \%$ & 3 & $27.27 \%$ & 3 & $27.27 \%$ \\
Expanded Nodes & 125 & $100.00 \%$ & 116 & $92.80 \%$ & 6 & $4.80 \%$ & 23 & $18.40 \%$ \\
Nodes in Queue & 293 & $100.00 \%$ & 286 & $97.61 \%$ & 15 & $5.12 \%$ & 55 & $18.77 \%$ \\
\hline
\end{tabular}

\subsubsection{Multiple Diagnoses}

This experiment evaluates $\mathrm{BFA}^{*}, \mathrm{CDA}^{*}$ and $\operatorname{CCDGA}^{*}(\varepsilon=1.0 \mathrm{e}-2)$ in finding 3 diagnoses.

The diagnosis results of CCDGA* are shown in Figure 17 where the three diagnoses were: (1) sensor Sen3 was in stuck_less fault mode; (2) heater $h 32$ was in less_power fault mode and (3) heater 
h31 was in less_power fault mode (all other components in the three diagnoses were normal). All the three diagnoses could explain the observation. BFA* and $\mathrm{CDA}^{*}$ gave the same results, which were not shown in this paper to avoid redundancy.

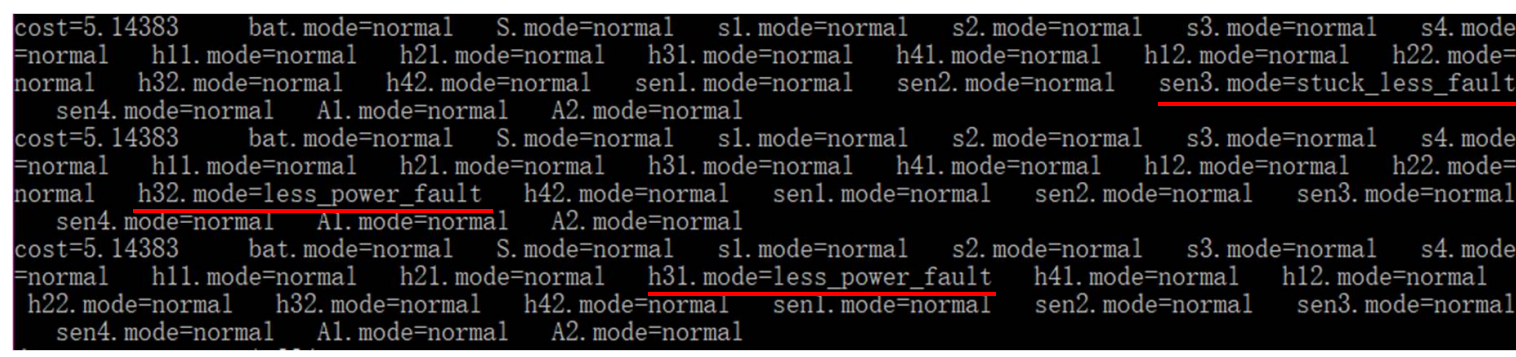

Figure 17. Diagnosis Results of CCDGA* for 3 diagnoses.

The diagnosis times for 10 experiments are given in Table 7, the average time is illustrated in Figure 18 and Table 8 shows the statistic information of nodes for search.

Table 7. 3-Diagnosis Time(s) for BFA*, $\mathrm{CDA}^{*}$ and CCDGA*.

\begin{tabular}{ccccccccccccc}
\hline Algorithm & $\mathbf{1}$ & $\mathbf{2}$ & $\mathbf{3}$ & $\mathbf{4}$ & $\mathbf{5}$ & $\mathbf{6}$ & $\mathbf{7}$ & $\mathbf{8}$ & $\mathbf{9}$ & $\mathbf{1 0}$ & Ave & Ratio \\
\hline BFA $^{*}$ & 2.326 & 2.234 & 2.228 & 2.179 & 2.146 & 2.152 & 2.060 & 2.227 & 2.122 & 2.163 & 2.18 & $100.00 \%$ \\
CDA $^{*}$ & 1.844 & 1.951 & 2.053 & 1.823 & 1.881 & 1.871 & 1.815 & 1.871 & 1.890 & 1.892 & 1.89 & $86.70 \%$ \\
CCDGA $^{*}$ & 1.347 & 1.593 & 1.465 & 1.642 & 1.534 & 1.517 & 1.477 & 1.496 & 1.482 & 1.524 & 1.52 & $69.72 \%$ \\
\hline
\end{tabular}

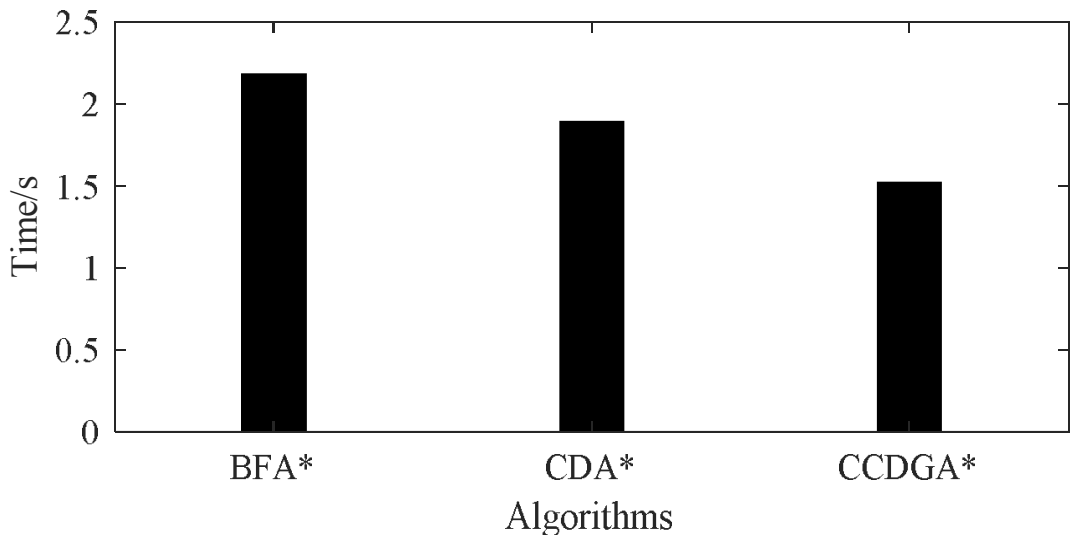

Figure 18. Average Diagnosis Time for BFA*, $\mathrm{CDA}^{*}$ and CCDGA*.

Table 8. Tried Candidates, Expanded Nodes and Nodes in Queue for Single Diagnosis.

\begin{tabular}{ccccccc}
\hline \multirow{2}{*}{ Algorithm } & \multicolumn{2}{c}{ BFA* $^{*}$} & \multicolumn{2}{c}{ CDA $^{*}$} & \multicolumn{2}{c}{ CDGA $^{*}$} \\
\cline { 2 - 7 } & Num & Ratio & Num & Ratio & Num & Ratio \\
\hline Tried Candidates & 35 & $100.00 \%$ & 14 & $40.00 \%$ & 9 & $25.71 \%$ \\
Expanded Nodes & 297 & $100.00 \%$ & 234 & $78.79 \%$ & 78 & $26.26 \%$ \\
Nodes in Queue & 689 & $100.00 \%$ & 610 & $88.53 \%$ & 190 & $27.58 \%$ \\
\hline
\end{tabular}

The statistical data in Tables 7 and 8 and Figure 18 are similar to the data for single-diagnosis.

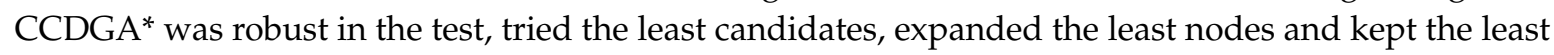
node in the queue. Although $\mathrm{CDA}^{*}$ also just tried a small number of candidates, the nodes expanded and reserved in the queue approaches the numbers of BFA*. In general, CCDGA* has both space and time advantages over $\mathrm{CDA}^{*}$ and $\mathrm{BFA}^{*}$. 
The section evaluates each module in the proposed methods comprehensively. Section 5.2 shows that MCMC-LTMS can not only check the consistency between system model, candidate and observation but also effectively find conflict and consistency set even though the novel LTMS does not explicitly utilize the system structure model. The two algorithms based on MCMC-LTMS are accessed in Section 5.3 where both single diagnosis and multiple diagnoses manifest that the proposed search methods can accurately skip improbable candidates based on the results from MCMC-LTMS. Compared with $\mathrm{BFA}^{*}$ and $\mathrm{CDA}^{*}$, both CCDLSA* and CCDGA* can significantly reduce the space and time cost in diagnosis.

\section{Conclusions and Future Work}

This paper presents research on reasoning and search in MBD for a strong-fault model. The greatest contribution is the proposition of consistency in MBD. In fact, the PC corresponds to the residual in Fault Detection and Identification (FDI) [11]. Evaluating a residual as 1 amounts to a $\mathrm{PC}$ is verified as a conflict. In the opposite, the PC is proven to be a consistency when the residual is 0 . The proposition of consistency makes MBD and FDI more consistent.

To obtain consistency when reasoning over discrete model, this paper proposed a novel MCMC-LTMS which is able to obtain multiple conflicts and consistencies in one reasoning process. In theory, MCMC-LTMS is complete for a strong-fault model and can cover all fault components in most cases. In the experiment, MCMC-LTMS found the only minimal conflict and all the maximal consistencies without using system structure information explicitly.

Faults can be isolated efficiently if the information in conflict and consistency is fully utilized. By Bayesian analysis and the introduction of missing alarm probability, a more exquisite probability model was proposed. Based on the probability model, $P$ (obs $\mid$ mode) can be evaluated more accurately.

An approximate version of $P($ obs $\mid$ mode $)$ provided by the novel probability model are used to construct the heuristic function for $\mathrm{A}^{*}$ search. With the proposed heuristic function, two $\mathrm{A}^{*}$ search algorithms are designed for single diagnosis and multiple diagnoses respectively. It has been proven theoretically that although the two algorithms may traverse the whole search space in the worst case, the diagnoses are correct and optimal for a strong-fault model. For the sharp priori distribution, both algorithms are robust except that when expecting too many diagnoses, CCDGA* is too time costly. The search algorithms may perform badly for the flat priori distribution. The experiment showed that all the tested algorithms were robust and could give the correct diagnoses but both CCDLSA* $^{*}$ and CCDGA* expanded fewer nodes in the search, tried fewer insignificant candidates and cost less time. Because CCDLSA* has a smaller search space than CCDGA*, CCDLSA* performed better in single diagnosis. Specifically, using BFA* as the criterion, for single diagnosis, CCDLSA* and CCDGA* reduced time by $33.33 \%$ and $28.74 \%$ respectively. However, CDA* just reduced by $3.45 \%$; for 3 diagnoses, CCDGA* reduced time by $30.28 \%$ and $\mathrm{CDA}^{*}$ only reduced by $13.3 \%$ as a comparison. Besides, CCDLSA* and CCDGA* also significantly outperformed CDA* in tried candidates, expanded nodes and nodes in queue, which indicated that the proposed algorithms cost less memory space.

Theoretical analysis and experiment manifest that the proposed diagnosis methods for a strong-fault model are effective and efficient. For a sharp priori distribution, both CCDLSA* and CCDGA $^{*}$ are suitable for single diagnosis but CCDLSA* is better. For multiple diagnoses, CCDGA* is effective with sharp priori distribution and a proper expected diagnosis number.

Currently, the search algorithms can only employ the consistencies found in the first iteration because the weights of nodes may change in the search tree based on new consistencies which causes reconstruction of the search tree. How to make use of consistencies incrementally will be studied in the future.

Author Contributions: Wenfeng Zhang conceived the idea, performed the experiments and wrote the paper; Qi Zhao and Hongbo Zhao designed the experiments; Gan Zhou and Wenquan Feng analyzed the data.

Conflicts of Interest: The authors declare no conflict of interest. 


\section{References}

1. Hwang, I.; Kim, S.; Kim, Y.; Seah, C.E. A survey of fault detection, isolation, and reconfiguration methods. IEEE Trans. Control Syst. Technol. 2010, 18, 636-653. [CrossRef]

2. Nica, I.; Pill, I.; Quaritsch, T.; Wotawa, F. The route to success-A performance comparison of diagnosis algorithms. IJCAI 2013, 13, 1039-1045.

3. Isermann, R. Fault-Diagnosis Systems: An Introductionfrom Fault Detection to Fault Tolerance; Springer: Berlin, Germany, 2006; ISBN 978-3-540-24112-6.

4. Gao, Z.; Cecati, C.; Ding, S.X. A Survey of Fault Diagnosis and Fault-Tolerant Techniques Part I: Fault Diagnosis. IEEE Trans. Ind. Electron. 2015, 62, 3757-3767. [CrossRef]

5. Gao, Z.; Cecati, C.; Ding, S.X. A survey of fault diagnosis and fault-tolerant techniques-part II: Fault diagnosis with knowledge-based and hybrid/active approaches. IEEE Trans. Ind. Electron. 2015, 62, 3768-3774. [CrossRef]

6. Venkatasubramanian, V.; Rengaswamy, R.; Yin, K.; Kavuri, S.N. A review of process fault detection and diagnosis Part I: Quantitative model-based methods. Comput. Chem. Eng. 2003, 27, 293-311. [CrossRef]

7. Venkatasubramanian, V.; Rengaswamy, R.; Ka, S.N. A review of process fault detection and diagnosis Part II: Qualitative models and search strategies. Comput. Chem. Eng. 2003, 27, 313-326. [CrossRef]

8. Venkatasubramanian, V.; Rengaswamy, R.; Kavuri, S.N.; Yin, K. A review of process fault detection and diagnosis part III: Process history based methods. Comput. Chem. Eng. 2003, 27, 327-346. [CrossRef]

9. Reiter, R. A theory of diagnosis from first principles. Artif. Intell. 1987, 32, 57-95. [CrossRef]

10. Wang, D.; Yu, M.; Low, C.B.; Arogeti, S. Model-Based Health Monitoring of Hybrid Systems; Springer: New York, NY, USA, 2013; ISBN 978-1-4614-7368-8.

11. Ding, S.X. Model-Based Fault Diagnosis Techniques; Springer: London, UK, 2013; Volume 53, ISBN 9788578110796.

12. Qin, S.J. Survey on data-driven industrial process monitoring and diagnosis. Annu. Rev. Control 2012, 36, 220-234. [CrossRef]

13. Ding, S.X. Data-Driven Design of Fault Diagnosis and Fault-Tolerant Control Systems; Springer: London, UK, 2014; ISBN 978-1-4471-6409-8.

14. De Kleer, J.; Williams, B.C.; De Kleer, J. Diagnosis with behavioral modes. IJCAI 1989, 89, 1324-1330.

15. De Kleer, J.; Williams, B.C. Diagnosing multiple faults. Artif. Intell. 1987, 32, 97-130. [CrossRef]

16. Doyle, J. A Truth Maintenance System. Read. Artif. Intell. 1981, 496-516. [CrossRef]

17. De Kleer, J. An assumption-based TMS. Artif. Intell. 1986, 28, 127-162. [CrossRef]

18. Williams, B.C.; Nayak, P.P. A Model-based Approach to Reactive Self-Configuring Systems. In Proceedings of the National Conference on Artificial Intelligence, Portland, OR, USA, 4-8 August 1996; pp. 274-282.

19. Feldman, A. Approximation Algorithms for Model-Based Diagnosis; TU Delft: Delft, The Netherlands, 2010; ISBN 9789090250236.

20. Feldman, A.; Provan, G.; Van Gemund, A. Approximate model-based diagnosis using greedy stochastic search. J. Artif. Intell. Res. 2010, 38, 371-413. [CrossRef]

21. Kurien, J.; Nayak, P. Back to the Future with Consistency-Based Trajectory Tracking. Available online: http:/ / www.aaai.org/Papers / AAAI/2000/AAAI00-057.pdf (accessed on 27 March 2018).

22. Williams, B.C.; Ragno, R.J. Conflict-Directed $\mathrm{A}^{*}$ and Its Role in Model-Based Embedded Systems. Discret. Appl. Math. 2007, 155, 1562-1595. [CrossRef]

23. Forbus, K.D.; de Kleer, J. Building Problem Solvers (Artificial Intelligence); The MIT Press: London, UK, 1993; ISBN 0262061570.

24. Stern, R.; Kalech, M.; Feldman, A.; Provan, G. Exploring the Duality in Conflict-Directed Model-Based Diagnosis. In Proceedings of the Twenty-Sixth AAAI Conference on Artificial Intelligence, Toronto, ON, Canada, 22-26 July 2012; pp. 828-834.

25. Shchekotykhin, K.; Jannach, D.; Schmitz, T. MERGEXPLAIN: Fast computation of multiple conflicts for diagnosis. IJCAI 2015, 15, 3221-3228.

26. Shchekotykhin, K.; Jannach, D.; Schmitz, T. A divide-and-conquer-method for computing multiple conflicts for diagnosis. CEUR Workshop Proc. 2015, 1507, 3-10.

27. Junker, U. QuickXplain: Preferred Explanations and Relaxations for Over-Constrained Problems. In Proceedings of the Nineteenth AAAI Conference on Artificial Intelligence, San Jose, CA, USA, 25-29 July 2004; pp. 167-172. 
28. Darwiche, A. Decomposable Negation Normal Form. J. ACM 2001, 48, 608-647. [CrossRef]

29. Torta, G. Compact Representation of Diagnoses for Improving Efficiency in Model Based Diagnosis. Ph.D. Thesis, Universita di Torino, Torino, Italy, 2006.

30. Biere, A.H.; Heule, M.; van Maaren, H.; Walsh, T. Handbook of Satisfability; IOS: Amsterdam, The Netherlands, 2009; ISBN 978-1-58603-929-5.

31. Dechter, R. Constraint Processing; Morgan Kaufmann: Burlington, MA, USA, 2003; Volume 33, ISBN 978-1-55-860890-0.

32. Pulido, B.; González, C.A. Possible conflicts: A compilation technique for consistency-based diagnosis. IEEE Trans. Syst. Man Cybern. Part B Cybern. 2004, 34, 2192-2206. [CrossRef]

33. Russell, S.; Norvig, P. Artificial Intelligence a Modern Approach; Pearson Education: Upper Saddle River, NJ, USA, 2013; ISBN 9780136042594.

(C) 2018 by the authors. Licensee MDPI, Basel, Switzerland. This article is an open access article distributed under the terms and conditions of the Creative Commons Attribution (CC BY) license (http://creativecommons.org/licenses/by/4.0/). 